\title{
Formation and evolution of early-type galaxies
}

\section{Models with quasi-cosmological initial conditions}

\author{
E. Merlin and C. Chiosi
}

\author{
Department of Astronomy, University of Padova, Vicolo dell'Osservatorio 2, 35122 Padova, Italy \\ e-mail: [merlin;chiosi]@pd.astro.it
}

Received 7 November 2005 / Accepted 12 April 2006

\begin{abstract}
Aims. In this study, with the aid of $N$-Body simulations based on quasi-cosmological initial conditions, we have followed the formation and evolution of two early-type galaxies of different total mass, from their separation from global expansion of the Universe to their collapse to virialized structures, the formation of stars and subsequent nearly passive evolution.

Methods. Using the PD-TSPH $N$-Body code, we developed the simulations of two model galaxies in quasi-cosmological context. The cosmological background we have considered is the Standard CDM. The models are made of Dark and Baryonic Matter in standard cosmological proportions (9:1), and have significantly different initial total mass, i.e. $1.62 \times 10^{12} M_{\odot}(\mathrm{A})$ and $0.03 \times 10^{12} M_{\odot}(\mathrm{B})$. Particular care has been paid to the star formation process, heating and cooling of gas, and chemical enrichment.

Results. Star formation is completed within the first $3 \mathrm{Gyr}$ in model A, whereas it lasts longer up to about 4 Gyr in model B. The models are followed for a long period of time, i.e. $13 \mathrm{Gyr}$ (model A) and $5 \mathrm{Gyr}$ (model B): in any case, well beyond the stages of active star formation. The structural properties of the present-day models are in good agreement with current observations. The chemical properties, mean metallicity and metallicity gradients also agree with available observational data. Finally, conspicuous galactic winds are found to occur.

Conclusions. The models conform to the so-called revised monolithic scheme, because mergers of substructures have occurred very early in the galaxy life. Our results agree with those obtained in other similar recent studies, thus strengthening the idea that the revised monolithic scheme is the right trail to follow in the forest of galaxy formation and evolution.
\end{abstract}

Key words. galaxies: elliptical and lenticular, $\mathrm{cD}$ - galaxies: formation - galaxies: evolution - methods: $N$-body simulations

\section{Introduction}

One of the major challenges in modern astrophysics is to understand the origin and evolution of galaxies, the bright elliptical ones in particular. In a Universe dominated by Cold Dark Matter (CDM) and, as recent observations seem to suggest, by some kind of Dark Energy in the form of a non-zero Cosmological Constant $\Lambda$, and containing a suitable mix of baryons and photons, cosmic structures are formed by the gravitational collapse of Dark Matter and are organized in a hierarchy of complexes (halos) inside which baryons dissipate their energy and collapse to form luminous systems. In this context, modelling the formation of early-type galaxies with simulations taking into account the dynamics of Dark Matter halos and gas, radiative cooling of baryons, star formation, and gas loss by galactic winds can be reduced to following schemes (Peebles 2002; Schade et al. 1999).

(1) The monolithic scenario of galaxy formation supposes and predicts that all early-type galaxies form at high redshift by rapid collapse and undergo a single prominent star formation episode (e.g. Eggen et al. 1962; Larson 1975; Arimoto \& Yoshii 1987; Bressan et al. 1994) ever since followed by quiescence. In favour of this scheme are the observational data that convincingly hint for old and homogeneous stellar populations (see Chiosi 2000, for a recent review of the subject). It is worth mentioning, however, that Kauffmann et al. (1993) and Barger et al. (1999) argue for the presence of recent evolution in the stellar populations of elliptical galaxies.
(2) The hierarchical scenario suggests instead that massive galaxies are the end product of subsequent mergers of smaller subunits, over time scales almost equal to the Hubble time in the particular cosmological model used; as the look back time increases, the density in comoving space of bright (massive) elliptical galaxies should decrease by a factor 2 to 3 (e.g. White \& Rees 1978; Kauffmann et al. 1993). In favour of this view are some observational evidences that the merger rate likely increases with $\sim(1+z)^{3}$ (Patton et al. 1997) together with some hint for a color-structure relationship for E \& S0 galaxies: the color becomes bluer at increasing complexity of a galaxy structure. This could indicate some star formation associated to the merger event. Finally, there are the many successful numerical simulations of galaxy encounters, mergers and interactions (e.g. Barnes \& Hernquist 1996). Nevertheless, contrary to the expectation from this model, the number density of elliptical does not seem to decrease with the redshift, at least up to $z \simeq 1$ (Im et al. 1996).

(3) There is a third scheme named dry merger view, in which bright ellipticals form by encounters of quiescent, no star forming galaxies. This view is advocated by Bell et al. (2004) who finds that the $B$-band luminosity density of the red peak in the colour distribution of galaxies shows mild evolution starting from $z \simeq 1$. As old stellar populations would fade by a factor 2 or 3 in this time interval, and the red colour of the peak tells us that new stars are not being formed in old galaxies, he argues that this mild evolution hints for a growth in the stellar mass of the red sequence, either coming from the blue-peak galaxies in 
which star formation is truncated by some physical process, or by "dry mergers" of smaller red, gas-poor galaxies.

(4) Finally, a fourth hybrid scenario named revised monolithic has been proposed by Schade et al. (1999), who suggests that a great deal of the stars in massive galaxies are formed very early-on at high $z$ and the remaining few ones at lower $z$. The revised monolithic ought to be preferred to the classical monolithic, as some evidences of star formation at $0.2 \leq z \leq 2$ can be inferred from the presence of the emission line of [OII], and also as the number frequency of early-type galaxies up to $z \simeq 1$ seems to be nearly constant.

However, a sharp distinction among the various scenarios could not exist in reality. As pointed out by Longhetti et al. (2000) early-type galaxies in isolation and in interaction (such as pair and shell galaxies) share the same distribution in diagnostic planes such as $H \beta$ vs. [MgFe], the classical tool to infer age differences. This means that secondary episodes of star formation may not only occur in merging (dynamically interacting) galaxies, but also in the isolated ones because of internal processes, thus supporting the Schade revised monolithic scheme. Captures (mergers) of small satellites by a galaxy born in isolation according to the monolithic scheme are reasonably possible (e.g. the Milky Way which is currently capturing the Sagittarius dwarf galaxy). In any case, as thoroughly discussed by Chiosi (2000), Chiosi \& Carraro (2002), Tantalo \& Chiosi (2004a) and Tantalo \& Chiosi (2004b), the age and intensity of the last episode of star formation (measured by the fractionary mass engaged in newly formed stars) cannot exceed some stringent limits, a few per cent and about one third of the Hubble time even in a massive elliptical, otherwise the typical broad band colours cannot be matched; they would be too blue compared to observations.

In addition to all this, elliptical galaxies are known to obey to a colour-magnitude relation (Bower et al. 1992): the colours become redder at increasing luminosity (and hence mass) of galaxies. The relation is tight for cluster galaxies and more disperse for field galaxies. The colour of a galaxy simply mirrors the colours of the constituent stellar populations. It can be shown that the colour-magnitude relation stems from a massmetallicity sequence and not an age sequence, see for instance Kodama \& Arimoto (1997) and Chiosi \& Carraro (2002) and references therein.

Long ago, Larson (1975) advocated the supernova driven galactic wind model to explain the colour-magnitude relation. He argued that massive galaxies retain gas and form stars for longer periods of time, and become richer in metals than the lowmass ones. In any case the star forming period even in the most massive galaxies seemed to be confined at very early epochs (say within the first Gyr to be generous). This kind of model has been extensively used to describe and predict the chemo-spectrophotometric properties of elliptical galaxies (e.g. Bressan et al. 1994, 1996; Tantalo et al. 1996, 1998; Chiosi et al. 1998). The short duration of the dominant star forming period has also been suggested by Bower et al. (1992) on the basis of very simple, empirical arguments: the vast majority of stars are formed at $z>2$ and subsequent activity if any should have occurred at $z \geq 0.5$.

Over the years, the situations became more intrigued because (i) the Larson (1975) model got in contrast with the observational constraint that massive elliptical have on the average $[\alpha / \mathrm{Fe}]$ ratios higher (super-solar) than the low mass ones (solar or below solar), thus implying the opposite trend for the duration of the star forming period as amply discussed by Chiosi et al. (1998), Chiosi (2000) and Chiosi \& Carraro (2002); (ii) of the results from absorption line indices, which seem to indicate that large age difference could exist. According to
Bressan et al. (1996), Tantalo et al. (1998), Tantalo \& Chiosi (2002, 2004a,b), the following picture emerges: all elliptical galaxies have been formed far back in the past, but have undergone different histories of star formation. Massive ellipticals had a single burst-like episode of stellar activity ever since followed by passive evolution, whereas the low mass ones stretched their star formation history over long periods of time in a series of bursts. This view has been supported by the $N$-Body simulations of Chiosi \& Carraro (2002) who find that the duration and mode of star formation is driven by the total mass (Dark plus Baryonic Matter) and/or the initial density of the galaxy. In addition to this the Chiosi \& Carraro (2002) models while predicting copious galactic winds much improved upon the simple-minded scheme by Larson (1975) and ruled out the point of inconsistency with the abundance ratios. We will came back to these issue later on. It is also supported by the most recent Spitzer-data (and SIRTF) in the far infrared, which have revealed the existence of very massive galaxies already in place at redshift $z \sim 6$; in addition to data HST observations have also brought into evidence galaxies in place at $z \sim 7-8$ (Bouwens et al. 2004).

All this can be understood within a model ruled by violent relaxation taking place in the remote past thanks to which clusters of just born stars can merge together into even denser clusters (e.g. Macchetto et al. 1996). In this context it is also worth mentioning that according to Bundy et al. (2005) (i) the mass of star forming galaxies increases with redshift ("downsizing") thus strongly weakening the hierarchical scenario as the main mechanism for forming giant ellipticals; and (ii) even if "dry mergers" clearly occur (van Dokkum 2005), they cannot play an important role in the assembling history of massive quiescent galaxies as the expected number density of massive galaxies at low redshift does not agree with observational data. Furthermore the massdownsizing of star forming galaxies seems to be almost independent of the environmental density, whereas one would expect a strong density dependence in the dry merger rate. Therefore, either classical or revised monolithic scenarios seem to be the right frame for the galaxy formation mechanism (Peebles 2002; Schade et al. 1999).

Basing on the pioneer studies by Katz (1991) and Katz \& Gunn (1991), and later by many authors among whom we recall Kawata (1999, 2001a,b); Kawata \& Gibson (2003) and Kobayashi (2005), we present numerical simulations carried out with the PD-TSPH code (Carraro et al. 1998) in order to investigate the formation mechanism on galactic scales. The scenario we have in mind and we intend to prove with the present simulations is the following one. At a certain (high) redshift a massive perturbation made of Dark Matter and baryons detaches from the Hubble flow and collapses on its own. It becomes a massive proto-galaxy, rich of substructures which moving inside the common gravitational potential well merge, form stars and eventually give rise to a single entity (the galaxy). The process is complete at high redshift (say before $z \simeq 2$ ) and from now on the galaxy evolves in "isolation"; subsequent captures of small satellites are possible without significantly altering the overall structure and evolution. In the case of a low-mass perturbation the stellar activity is prolonged over long time scales. Mergers between two galaxies may occur but they are a sort of rare, spectacular event and not the basic mechanism for assembling massive ellipticals.

The plan of the paper is as follows. Section 2 presents the procedures we have used to derive the initial conditions for our models; the procedure we have adopted is outlined and compared with other techniques available in literature. Section 3 describes some assumptions we have made for some fundamental 
physical processes that are included in the numerical simulations, namely cooling, star formation and initial mass functions, heating and chemical enrichment, and shortly outlines the numerical implementation of these processes in the $N$-Body PD-TSPH code developed by the Padova group over the years, together with a brief description of the probabilistic view of star formation, heating, cooling and chemical enrichment proposed by Lia et al. (2002). Section 4 presents the key parameters of the models we plan to calculate, and in particular it deals with the initial conditions derived from the method described in Sect. 2. Section 5 presents the results: the spatial evolution, the star formation history, the energy balance, the chemical enrichment and other details. Finally, Sect. 6 contains some concluding remarks.

\section{Search of the initial conditions}

The choice of the initial conditions is perhaps the fundamental step on which much of the future evolution of structures (galaxies) will depend. As noted by Power et al. (2003), despite the popularity of cosmological $N$-body simulations in the last years, there is very little detail in literature about setting up initial conditions.

The scheme we have adopted can be defined as "quasicosmological". It starts from realistic simulations of the cosmological evolution of the Universe that are suitably adapted to the numerical simulations of galaxy formation and evolution.

\subsection{Quasi-cosmological initial conditions}

Our initial conditions stem from the studies of Katz (1991), Katz \& Gunn (1991) and Kawata (1999). We adopt the Standard Cold Dark Matter Universe with $\Omega(z)=\Omega_{0}$, the matter density at the present time. Therefore, the basic parameters of the model are $H_{0}, \Omega_{0}$, and $\sigma_{8}$ (the rms mass fluctuations of the present-day Universe that normalizes the density fluctuations and fix the initial redshift of the simulations via the amplitude of the density fluctuations $)^{1}$. These models do not consider the presence of the Cosmological Constant $\Lambda$ as indicated by the recent W-MAP data (Bennett et al. 2003).

\subsubsection{Preparing the quasi-cosmological models}

To derive the initial conditions (positions and velocity field) of Dark and Baryonic Matter particles of the pro-galaxy, we start from simulating an initial large grid representing the Universe using the public software GRAFIC 2 by Bertschinger (2001). The initial Universe grid is a very large cube with $\sim 40$ comoving Mpc per side. In the grid we select a peak of over-density which is assumed as the centre (or very close to it) of a new cubic subgrid of smaller size. In practice, we fix the size of the sub-grid looking for the distance from the peak at which the over-density goes to zero. This distance is taken as half the size of the cube dimension $l$. In this sub-grid both Dark and Baryonic Matter are present. Their respective barycentres do not necessarily coincide each other nor are both coincident with the geometrical centre of the cube. Then we isolate this cubic volume, and using GRAFIC2 multi-level initial conditions generator we improve upon its resolution, thus describing small-scale perturbations in great detail, superposed to the large scale perturbations described in the firstlevel grid. Subsequently, using the radial distance of each particle (Dark Matter and Baryons) from the centre of the cube, we

1 All equations used in this section are from Padmanabhan (1993) unless otherwise specified. select the sample of particles contained in a sphere with radius equal to $l / 2$. The volume of the sphere is about half of the volume of the cube and accordingly the number of particles in the sphere is about half of the number of particles in the cube. It is worth recalling that, due to the cosmological perturbations, as the baryons in the cube could have a slight offset with respect to Dark Matter, the same could happen with the spheres of baryons and Dark matter. Therefore, the numbers of particles of the two matter species can be slightly different, and not be exactly concentric. This however is less of a problem, because first it is physically understandable (perturbations of the density field and infall of baryons in the Dark Matter potential wells), and second the difference in the numbers of particles is always small. By replacing the cube with a sphere we avoid spurious tidal effects on the particles. The sphere represents our proto-galaxy in very early stages. It is worth noticing that centering the new coordinate system at the geometrical centre of the cube rather than at the mass-weighed centre leads to the possibility that the peak of the density perturbation does not coincide with the centre of the sphere. The particle positions are then referred to the new system: the distances provided by GRAFIC 2 in comoving Mpc are translated to proper distances in Mpc according to

$d_{\text {pr }}=a_{\text {start }} \times d_{\text {comov }}$

where $a_{\text {start }}=\left(1+z_{\text {start }}\right)^{-1}$ is the value of the expansion factor at the initial redshift, $d_{\text {comov }}$ is the distance in the comoving frame of reference and $d_{\mathrm{pr}}$ is the proper distance. The velocities are then referred to the centre of the sphere, dropping the motion of the sphere as a whole and leaving to each particle only the peculiar motion. To each particle we add the velocity of the Hubble flow, which is simply given by

$v_{\text {flow }}=H\left(z_{\text {start }}\right) \times d_{\text {pr }}$

(this velocity is radially oriented). Note that in order to calculate $H(z)$ one has to assume a cosmological model. In the Standard CDM cosmology

$H(z)=H_{0} \sqrt{\Omega_{\mathrm{m}}(1+z)^{3}}$.

Finally we add a certain amount of rigid rotation. The associated spin parameter $\lambda$ is

$\lambda=\frac{J|E|^{1 / 2}}{G M^{5 / 2}}$

where $J$ is the angular momentum, $E$ is the initial binding energy, and $M$ the total mass of the system. Typical values of $\lambda$ range from 0.02 and 0.08 (White 1984), which corresponds to angular velocities of the order of fractions of a complete rotation over time-scales as long as about ten free-fall time-scales (Carraro et al. 1998). As the free-fall time-scale depends on the density according to

$t_{\mathrm{ff}}=\frac{1}{\sqrt{4 \pi G \rho}}$

it strongly depends also on the initial redshift. The background density for matter scales indeed as $(1+z)^{3}$. Introducing the rigid rotation somehow simulates and takes into account the effects of fluctuations with wavelength longer than the typical size of the proto-galaxy (e.g. Kawata 1999); neglecting rotation would imply that the matter surrounding the proto-galaxy has no effect on its evolution. 


\subsubsection{Densities and masses: some cautionary remarks}

The theory of spherical collapse (cf. Padmanabhan 1993) would allow us to estimate the approximate redshift at which turnaround and collapse of an homogeneous and expanding sphere should occur under the action of self-gravitation. The relations of interest here are

$$
\begin{aligned}
& 1+z_{\text {turn }}=0.57\left(1+z_{i}\right) \delta_{i} \\
& 1+z_{\text {coll }}=0.36\left(1+z_{i}\right) \delta_{i}
\end{aligned}
$$

where $\delta_{i}$ is the mean over-density of the sphere (proto-galaxy) at the initial redshift. But these values are mere approximations of reality.

First of all, the classical theory of spherical collapse stands on top-hat perturbations, in which the particle velocity field is determined only by the Hubble flow. The presence of peculiar velocities and density perturbations strongly alters the predictions of this simple theory. If the initial conditions are such that there is a single, strong peak in the density field, a more reliable model could be the secondary infall model (e.g. Miller \& Bertschinger 1986). Models for the spherical collapse taking the initial kinetic energy of peculiar motions into account do actually exist, see e.g. Padmanabhan (1993). As these models go beyond our aims, they will not be consider here. Second, cutting the sphere out of the cosmological Universe-grid and isolating it in a void space implies that the mean density of the Universe coincides with the mean density of the sphere. Therefore, assigning a certain over-density to the sphere will force it to follow an evolutionary path typical of a Universe with $\Omega>1$. This means that it will collapse on a faster time-scale. In cosmological simulations, the problem is nowadays solved using periodic boundary conditions. To cope with this point of uncertainty in our models, we have limited ourselves to consider only low initial over-densities $(\leq 0.1)$.

Furthermore, even if an univocal relationship should exist between redshift and proto-galaxy over-density, in reality fluctuations around the mean value of $\delta$ are possible, the fluctuations being larger at decreasing mass of the proto-galaxy.

Finally, the spherical symmetry and sharp confinement of matter in the sphere we have adopted to avoid complications due to possible irregular shapes of the proto-galaxy, imply that particles in the outskirts of the galaxy will meet sudden vanishing of density and velocity at the surface of the sphere.

Given all these uncertainties, it is clear that the results of the simulations will be only loosely related to theoretical predictions such as expected collapse redshifts.

Considering the total mass as a free parameter would allow to investigate a wider range of possibilities, but such a choice would somehow weaken the above quasi-cosmological scheme and the peculiar velocities (which depend on the overdensity) should be properly adjusted. Therefore we prefer to assign the sphere the mass given by the "correct" value of the mean overdensity output by GRAFIC2.

\subsection{Comparison with other initial conditions}

It is worth commenting here the main difference between our initial conditions and those adopted by Carraro et al. (1998) and Chiosi \& Carraro (2002). They start with a spherical model containing Dark and Baryonic Matter with masses in the standard proportions 9:1. They set the initial density profile for the Dark Matter component to be

$\rho(r)=\rho_{\mathrm{c}} \frac{r_{\mathrm{c}}}{r}$ where $r_{\mathrm{c}}$ and $\rho_{\mathrm{c}}$ are radius and density of a small central sphere. It resembles the well-known Navarro et al. (1996) law (see below) in the central regions. This indeed, independently of cosmological models, initial spectrum of perturbations and total mass, is $\propto r^{-1}$ in the central regions and $\propto r^{-3}$ in the external ones.

The spatial positions of Dark Matter particles are obtained by MonteCarlo deviations from the distribution law. The initial velocities of Dark Matter particles are obtained from the velocity dispersion $\sigma(r)$ assuming equi-partition among the three components, i.e.

$v(r)=\frac{1}{3} \sigma(r)=\sqrt{\rho_{\mathrm{c}} r_{\mathrm{c}} G r \ln \left(\frac{R_{T}}{r}\right)}$

which is obtained by inserting Eq. (7) in

$\rho(r) \sigma(r)^{2}=\int_{r}^{R_{T}} \frac{G M\left(r^{\prime}\right)}{r^{\prime 2}} \rho\left(r^{\prime}\right) \mathrm{d} r^{\prime}$

(Binney \& Tremaine 1987).

The gas particles (baryons) are then homogeneously and randomly distributed in the spherical halo of cold Dark Matter with null velocity field to simulate the infall of primordial gas into the potential well of Dark Matter (White \& Rees 1978).

In practice, this is equivalent to start with a halo of Dark Matter already detached from the Hubble flow which begins to collapse carrying along the baryonic matter. Even if all this sounds physically reasonable, these initial conditions already contain the solution of the problem: a self-gravitating, collapsing halo of Dark Matter whose density profile already resembles the final structure one is looking at. Moreover, in the CDM cosmology catching up of baryons by the potential wells of Dark Matter happens much earlier than the time at which a proto-galactic halo detaches from the Hubble flow.

The initial radius is set to be equal to the virial radius $R_{200}$ of the proto-galaxies, which is defined as the radius of a sphere with mean density equal to 200 times the mean background density of the Universe, $\rho_{u}$, which in the case of a Standard CDM model $\left(\Omega_{0}=1\right)$ is given by

$\rho_{u}(z)=\frac{3 H_{0}^{2}}{8 \pi G}(1+z)^{3}$

with obvious meaning of the symbols, since the minimum density required for collapse is about $200 \rho_{u}(z)$ (Navarro et al. 1996; Padmanabhan 1993). Finally, star formation is supposed to occur only after the virialization of the CDM halo.

To summarize, the present initial conditions differ from those used in past similar studies carried out by the Padua group with the same software (e.g. Chiosi \& Carraro 2002) at least in the three aspects:

- The initial velocities taking into account the peculiar motions and the Hubble flow provide a more realistic description of the complexity of the galaxy formation process as compared to the "static" model.

- The variation of the perturbation amplitude and spectrum allows us to better explore their effects on the resulting galaxy models and properties.

- The peculiar velocities are set to their initial value in a selfconsistent way securing the correct growth of the perturbations on all scales of interest (e.g. Katz 1991).

Modern numerical cosmological simulations include more sophisticated methods, such as evolution in comoving space and resimulation at different degrees of accuracy and resolution 
(see, e.g. Kawata \& Gibson 2003). The present scheme has to be intended as the initial step towards future improvements. Nevertheless we are confident that the present method, despite its limitations, is reasonably suited to study the formation and the morphological, dynamical and chemical evolution of earlytype galaxies as isolated objects.

\section{Basic physics in galaxy models}

In this section we shortly summarize the basic physical processes that are thought to lead galactic formation and evolution, together with some details on their implementation in the fully Lagrangian PD-TSPH. More details on the code can be found in Carraro et al. (1998), Buonomo et al. (2000), Lia et al. (2002) and Chiosi \& Carraro (2002).

Key physical ingredients ingredients of the whole problem are the gravitational force that acts on all kinds of particle and is implemented by the classical Tree-code of Barnes \& Hut (1986) with quadrupole expansion, and the hydrodynamic processes, which instead act only on the energy and motions of the gas particles together with star formation, chemical evolution, gas cooling by radiative processes, and finally gas heating by energy injection from supernova explosions, stellar wind, UV cosmic radiation and others.

The system of gas particles can be viewed as a collisional fluid governed by the classical equations, which in Lagrangian notation are

$$
\begin{aligned}
\frac{\mathrm{d} \rho}{\mathrm{d} t} & =-\rho \nabla \vec{v} \\
\frac{\mathrm{d} v}{\mathrm{~d} t} & =-\frac{1}{\rho} \nabla P-\nabla \Phi \\
\frac{\mathrm{d} u}{\mathrm{~d} t} & =-\frac{P}{\rho} \nabla u+S \\
\nabla^{2} \Phi & =4 \pi G \rho
\end{aligned}
$$

i.e. the continuity or mass conservation equation, the Euler or momentum conservation equation, the energy conservation equation, and the Poisson equation linking the gravitational potential to the medium density, respectively. The companion equation of state is that of a perfect gas $P=\frac{R \rho T}{\mu}$.

Gas-dynamics is described with the $S P H$ formalism by Monaghan \& Lattanzio (1985) and Hernquist \& Katz (1989), as implemented by Carraro et al. (1998), Buonomo et al. (2000), Lia et al. (2002) and Chiosi \& Carraro (2002); in particular, a probabilistic approach to non-adiabatic processes such as star formation and gas restitution is used (see below). The term $S$ in Eq. (13) is the energy source (otherwise known as source function) from all heating and cooling processes. It is written as

$S=\frac{\Lambda-\Gamma}{\rho}$

where $\Lambda$ and $\Gamma$ are the cooling and heating rates, respectively (see Sects. 3.1 and 3.3 for details).

To derive the mean value of any physical quantity $f(r)$ over a certain interval we adopt the so-called "gather/scatter" functional relation suggested by Hernquist \& Katz (1989):

$\langle f(r)\rangle=\int f\left(r^{\prime}\right) \frac{1}{2}\left\{W\left(r-r^{\prime}, h\left(r^{\prime}\right)\right)+W\left(r-r^{\prime}, h(r)\right)\right\} \mathrm{d} r^{\prime}$,

where $h$ is the smoothing length, which gives the extension of the volumes over which a suitable space average is made. For the kernel $W(r)$ we adopt the form proposed by Monaghan \& Lattanzio (1985)

$$
W(r, h)=\frac{1}{\pi h^{3}}\left\{\begin{array}{l}
1-\frac{3}{2}\left(\frac{r}{h}\right)^{2}+\frac{3}{4}\left(\frac{r}{h}\right)^{3} \text { if } 0 \leq \frac{r}{h} \leq 1 \\
\frac{1}{4}\left(2-\left(\frac{r}{h}\right)\right)^{3} \text { if } 1 \leq \frac{r}{h} \leq 2 \\
0 \text { if } \frac{r}{h} \geq 2
\end{array}\right.
$$

As usual, in the Euler Eq. (12) an additional viscosity term is introduced to describe more complicated processes than simple shock waves. There are many possible formulations for this additional term, among which we adopt the one by Monaghan \& Lattanzio (1985)

$$
\prod_{i j}=\frac{-\alpha \mu_{i j} \overline{c_{i j}}+\beta \mu_{i j}^{2}}{\overline{\rho_{i j}}} \overline{f_{i j}}
$$

where

$\mu_{i j}=\left\{\begin{array}{l}\frac{v_{i j} r_{i j}}{h_{i j}\left(r_{i j}^{2} / h_{i j}^{2}+\eta^{2}\right)} \quad \text { if } v_{i j} r_{i j}<0 \\ 0 \quad \text { if } v_{i j} r_{i j} \geq 0\end{array}\right.$

with the following meaning of the symbols: $v_{i j}=v_{j}-v_{i}, \bar{c}_{i j}=$ $\left(c_{j}+c_{i}\right) / 2$ is the mean sound velocity; $h_{i j}=\left(h_{j}+h_{i}\right) / 2, \bar{\rho}_{i j}=$ $\left(\rho_{j}+\rho_{i}\right) / 2, \alpha, \beta$ and $\eta$ are constant coefficients with typical values $\sim 1, \sim 2$, and $\sim 0.1$, respectively (the latter coefficient is inserted only to prevent numerical divergences); and $\overline{f_{i j}}$ represents a shear correcting term to the artificial viscosity (cf. Steinmetz 1996).

The code calculates the time-step of each individual gas particle by means of the Courant condition,

$$
\Delta t_{C, i}=\frac{C h_{i}}{\left[h_{i}\left|\nabla v_{i}\right|+c_{i}+1.2\left(\alpha c_{i}+\beta_{m} a x_{j}\left|\mu_{i j}\right|\right)\right]}
$$

where $C$ is a constant nearly equal to $\sim 0.3$. For all kinds of particle (both collisional and collisionless) in presence of gravity a more stringent condition is required (Katz et al. 1996)

$\Delta t_{G, i}=\eta \times \min \left[\frac{\eta \epsilon}{|v|},\left(\frac{\epsilon}{|a|}\right)^{1 / 2}\right]$

where $\epsilon$ is the softening parameter and $\eta$ is constant with value $\sim 0.5$. Finally, the time-step for the $i$ th particle is the least of the two: $\Delta t_{i}=\min \left(\Delta t_{C, i}, \Delta t_{G, i}\right)$; and the time step for the whole system is taken to be the smallest of all particles time steps.

Furthermore, the smoothing length is varied with time and space according to the prescription by Benz (1990)

$\frac{\mathrm{d} h}{\mathrm{~d} t}=-\frac{1}{3} h \nabla v$.

The softening parameters we have adopted for the different components of our simulations are given in Table 2.

\subsection{Cooling}

Gas cooling by many radiative processes perhaps plays the dominant role in the collapse of baryonic matter in galaxies and star formation, since it is the comparison between the cooling and the dynamical time scales, respectively given by

$t_{\text {cool }}=\frac{E}{\dot{E}} \simeq \frac{3 \rho k T}{2 \mu \Lambda(T)} \quad$ and $\quad t_{\text {dyn }} \simeq \frac{\pi}{2} \sqrt{\frac{2 G M}{R^{3}}}$

(where $\rho$ is the mean gas density and $\Lambda(T)$ is the cooling rate of the gas at the temperature $T$ ) that decides whether the gas 
will collapse nearly on the free-fall time (if $t_{\text {cool }}<t_{\text {dyn }}$ ) or just contract through subsequent equilibrium conditions (if $t_{\text {cool }}>$ $\left.t_{\text {dyn }}\right)$.

Many cooling processes are known. In general, the radiative cooling depends on density, chemical composition and temperature (from this latter very strongly). For $T>10^{4} \mathrm{~K}$ the most efficient process is bremsstrahlung emission from the ionised plasma. In the range $100 \leq T \leq 10^{4} \mathrm{~K}$ collision between $\mathrm{H}_{2}$ molecule and/or $\mathrm{H}$ atoms dominate via rotational and vibrational energy decay. Finally, for $T<100 \mathrm{~K}$, the dominant contribution comes from CO molecules. See Chiosi et al. (1998) for more details on the subject.

Another important cooling mechanism is the inverse Compton effect, which is particularly important at high redshifts. The cooling rate of a gas with electron number density $n_{\mathrm{e}}$ and temperature $T$, in a field of radiation with density $\rho_{\mathrm{R}}$ and temperature $T_{\mathrm{R}}$, is

$\Lambda_{\text {comp }}=\frac{4 \sigma_{\mathrm{T}} n_{\mathrm{e}} \rho_{\mathrm{R}}\left(T-T_{\mathrm{R}}\right)}{m_{\mathrm{e}}}$.

Estimating the cooling time for matter immersed in the CMB we obtain

$t_{\text {comp }}=\frac{\left[3 m_{\mathrm{p}} m_{\mathrm{e}}(1+z)^{-4}\right]}{\left(8 \mu \sigma_{\mathrm{T}} \Omega_{\mathrm{R}} \rho_{\text {crit }}\right)}$

which is nearly equal to the dynamical time for collapse redshifts greater than 7, independently of the galaxy mass. As our simulations begin at high redshift, the inverse Compton effect has been taken into account. In this paper we have adopted the cooling rates given by Chiosi et al. (1998, and references therein) who have amalgamated results from different sources and to whom the reader should refer for all details. The same cooling rate have been adopted by Carraro et al. (1998), Buonomo et al. (2000), Lia et al. (2002), and Chiosi \& Carraro (2002) in their studies of galaxy formation and evolution.

\subsection{Star formation rate and initial mass function}

Star formation is among the most complicate and poorly known physical aspects of astrophysics despite its relevance in many issues. From simple minded arguments, there are at least three prerequisites for a gas (molecular cloud) to be eligible to star formation: the gas has to be in convergent motion, i.e. the velocity divergence must be negative; the gas must be gravitationally unstable, i.e. it must satisfy the Jeans condition $\tau_{\text {sound }} \geq \tau_{\mathrm{ff}}$ (where $\tau_{\text {sound }}$ is the local sound velocity); the gas must be cooling, i.e. it has to verify the relation $\tau_{\text {cool }} \ll \tau_{\text {ff }}$ (see Sect. 3.1). Then, the rate of star formation (SFR) is customarily expressed by the Schmidt (1959) law

$\frac{\mathrm{d} \rho_{*}}{\mathrm{~d} t_{\mathrm{g}}}=-\frac{\mathrm{d} \rho_{\mathrm{g}}}{\mathrm{d} t_{\mathrm{g}}}=c^{*} \frac{\rho_{\mathrm{g}}}{t_{\mathrm{g}}}$

where $c^{*}$ is the so-called dimensionless efficiency of star formation, and $t_{\mathrm{g}}$ is a characteristic time scale. Normally, SPH codes treat star formation simply implementing the Schmidt law in the computational language and transforming part of gaseous particles which satisfy the three conditions above in new, collisionless particles of different mass ("stars"), thus giving rise to a huge increase in the total number of particles. In order to avoid this creation of new particles with different mass as a result of star formation, Lia et al. (2002) proposed a new interpretation of the Schmidt law: the star formation rate given by Eq. (25) was interpreted as the probability that at each time step a gas particle is instantaneously and fully turned into a star particle (thus losing its collisional properties). They showed that, averaged over a large fraction of the Hubble time, this probabilistic view of the star formation rate converges to the results of more conservative methods in which the masses of the newly formed stellar particles strictly follow the dictates of the chosen IMF law. The models we are going to present are based on this view of the star forming process.

Furthermore, even if Buonomo et al. (2000) argued that the condition on the velocity divergence of gas particles (negative) is not strictly required, the same arguments do not apply to our case because of the initial expansion phase of our proto-galaxies. Neglecting it, star formation could occur too early on.

Following Buonomo et al. (2000), we adopt $c^{*}=1.0$ for all the models. The characteristic time scale is chosen to be the maximum between $\tau_{\text {cool }}$ and $\tau_{\text {ff }}$ time-scales. Numerical experiments show that in most situations $t_{\mathrm{g}}=\tau_{\mathrm{ff}}$ is a good choice.

Chiosi \& Carraro (2002) have found that the star formation history (rate vs. time) depends on the depth of the gravitational potential well of a galaxy. In the case of deep potentials (such as in massive and/or dense galaxies), once star formation has started and energy is injected to the gas by supernova explosions, stellar wind, etc..., this is not enough to push the gas out of the potential well. A sort of balance between cooling and heating is reached and the gas consumption by star formation goes to completion. Star formation cannot stop until the remaining gas is so little that any further energy injection will eventually heat it up to such high energies (temperatures) that the gravitational potential is overwhelmed. No more gas is left over and star formation is quenched. The star formation history resembles a strong unique burst of activity, a sort of monolithic star forming event, taking place over a certain amount of time, of the order of 1 to 2 Gyr. In contrast, in a galaxy of low mass and/or density and hence shallow gravitational potential, even a small star forming activity will heat up the gas above the potential well. Some of it is soon lost in galactic wind, the remaining one becomes so hot that it will take long time to cool down and to form new stars. The cycle goes on many times in a sort of repeated bursting mode of star formation taking place during long periods of times if not for ever.

In each generation of stars, otherwise known as Single Stellar Population (SSP), the stellar masses are known to obey a distribution function, named Initial Mass Function (IMF), which is usually represented by a power law. The most popular of these functions is the one by Salpeter (1955). More recent ones and currently used in galaxy evolution models are those by Arimoto \& Yoshii (1987) and Kroupa (1998). The three IMFs are shortly summarized here:

$$
\begin{aligned}
& \Phi_{S}(M)=C_{S} M^{-1.35} \quad \text { Salpeter } \\
& \Phi_{K}(M)= \begin{cases}C_{K 1} M^{-0.5} & \text { if } M<0.5 \\
C_{K} M^{-1.2} & \text { if } 0.5<M<1 \quad \text { Kroupa } \\
C_{K} M^{-1.7} & \text { if } M>1\end{cases} \\
& \Phi_{A}(M)=C_{A} M^{-1.00} \quad \text { Arimoto \& Yoshii }
\end{aligned}
$$

where $C_{S}=0.1716, C_{K 1}=0.48, C_{K}=0.295$ and $C_{A}=0.145$. Needless to say, the IMF bears very much on many aspects of galactic evolution. In our models we have adopted the Kroupa (1998) IMF, originally designed to describe the situation for the solar vicinity. We prefer not to use the Arimoto \& Yoshii (1987) IMF, even if it was claimed to be best suited to early-type galaxies, because it would produce a too high metallicity (see below). 


\subsection{Heating}

Gas heating is due to supernova explosions, stellar winds and UV radiation emitted by massive stars, cosmic ultraviolet light, and to the cosmic background radiation. In addition to this we have several mechanisms of mechanical and dynamical nature which are already accounted for by the first term of the energy in Eq. (13).

The total heating rate by radiative processes is given by

$\Gamma=\frac{E_{\mathrm{SNI}}+E_{\mathrm{SNII}}+E_{w}+E_{\mathrm{UV}}}{\Delta t}$

where the terms refer to type Ia and type II supernovae, stellar winds and ultraviolet flux, respectively. In our model we consider only the supernova heating and neglect the other two. In brief, (i) the UV flux from massive stars is absorbed by the interstellar molecular gas and re-emitted in the far infrared and thus is soon lost by radiation; (ii) the kinetic contribution by stellar wind should be treated on much smaller scales than those permitted by the resolution of these simulations. This is a point to keep in mind because the energy budget from stellar winds from massive stars may parallel that by supernova explosions. Finally, we do not include the heating by UV cosmic radiation. This may be a point of weakness because this heating source could delay gas cooling and star formation in turn, and even stop star formation at later times by contrasting gas cooling. Although according to Navarro \& Steinmetz (1997) it can be neglected at least during the first stages of galaxy formation, careful investigation of this issue is mandatory to properly understand the mechanisms of star formation.

Supernova explosions return nuclearly processed gas to the interstellar medium, and heat it up. Type Ia supernovae from binary systems and type II supernovae from massive stars affect in a different way the heating of the interstellar medium because of their much different evolutionary time scales. The progenitors of type Ia are CO-white dwarf accreting mass from a companion, which in turn can be either a red giant or another CO-white dwarf. When and if the mass accreting white dwarf grows to the Chandrasekhar limit $1.4 M_{\odot}, \mathrm{C}$-ignition can take place inducing thermal runaway and consequent supernova explosion. The time scale for this to occur ranges from a few hundreds Myr to several Gyr. In contrast, type II supernovae generated by core collapse in massive stars have time scales of the order of a few Myr. Taking into account such different behaviour is an important aspect of modelling galaxy evolution. We assume that each supernova explosion liberates $k_{\mathrm{SN}} \times 10^{51}$ erg of energy; anyway, only a small fraction of which - say $10 \%$ - can be thought as given back to the ISM (Thornton et al. 1998); the parameter $k_{\mathrm{SN}}$ fixes the amount of energy which is actually made available to the gas and not dispersed by radiative processes. This energy injection increases the internal energy of the gas particles in the proximity of the explosion according to the SPH formalism and some kinematical/dynamical effects ought to be expected. However, as for stellar winds, it is difficult to correctly take into account the effect of this on the kinematics of the nearby gas particles, as the scales on which these effects should be noticeable are too small if compared to the resolution of our simulations. Therefore we simply give back the whole amount of energy to the thermal budget of the nearby particles. This approximation has little impact on the dynamical evolution of the whole system, due to the high cooling rates involved in the process (see e.g. Carraro et al. 1998; Kawata \& Gibson 2003).

To describe the rates of supernova explosions we adopt the popular formulation by Greggio \& Renzini (1983); see there and
Table 1. Initial parameters for models A and B in the Standard CMD cosmological background.

\begin{tabular}{lll}
\hline \hline Model & $\mathrm{A}$ & $\mathrm{B}$ \\
\hline Initial redshift & 50 & 53 \\
$\Omega_{\mathrm{m}}$ & 1 & 1 \\
$\Omega_{\Lambda}$ & 0 & 0 \\
$H_{0}=50 \mathrm{~km} \mathrm{Mpc}^{-1} \mathrm{~s}^{-1}$ & 50 & 50 \\
$\sigma_{8}$ & 0.5 & 0.5 \\
Spin parameter & 0.02 & 0.02 \\
Initial mean overdensity & 0.12 & 0.12 \\
IMF & Kroupa & Kroupa \\
\hline
\end{tabular}

Table 2. Initial dynamical and computational parameters for models A and $\mathrm{B}$.

\begin{tabular}{lll}
\hline \hline Model & $\mathrm{A}$ & $\mathrm{B}$ \\
\hline Initial number of gas particles & 13719 & 13904 \\
Initial number of CDM particles & 13685 & 13776 \\
Initial total mass $\left(10^{12} M_{\odot}\right)$ & 1.62 & 0.03 \\
Initial baryonic mass fraction & 0.10 & 0.10 \\
Initial radius (kpc) & 33 & 9 \\
Softening parameter for gas $(\mathrm{kpc})$ & 1 & 0.5 \\
Softening parameter for DM $(\mathrm{kpc})$ & 2 & 1 \\
\hline
\end{tabular}

references therein for details. To describe the energy feedback, we adopt once more the probabilistic description of Lia et al. (2002), to which we remand for a detailed description.

\subsection{Chemical enrichment}

The material, processed by nuclear reactions in stellar interiors and given back to the interstellar medium by supernova explosions and/or stellar winds, enriches the gas in metals. To correctly estimate the chemical enrichment, first one has to carefully calculate the stellar ejecta and the chemical yields per stellar generation, second to suitably spread the newly formed metals into the surrounding gas; see Lia et al. (2002) for a complete description of the physical processes involved in the computation and of their implementation in the code (the laws governing the gas restitution together with the chemical enrichment are treated in the same probabilistic manner as the ones governing the star formation and supernova rate processes).

The metals are distributed among the gas particles by means of the diffusion equation

$$
\frac{\mathrm{d} Z}{\mathrm{~d} t}=-\kappa \nabla^{2} Z
$$

where $\kappa$ is the diffusion coefficient, for which we adopt the estimate by Lia et al. (2002) $\kappa=9.25 \times 10^{16} \mathrm{~km}^{2} \mathrm{~s}^{-1}$ based on the Thornton et al. (1998) models. In our simulations we explicitly follow the contribution to the mass abundance of ten elements, namely $\mathrm{Z}$ (metals in general), $\mathrm{He}, \mathrm{C}, \mathrm{O}, \mathrm{N}, \mathrm{Mg}, \mathrm{Si}, \mathrm{S}, \mathrm{Ca}$ and $\mathrm{Fe}$.

\section{Galaxy models: the parameters}

\subsection{The cosmological initial conditions}

As already pointed out, in this paper we present two simulations, whose parameters are summarized in Table 1.

All simulations are framed in a flat Universe with spectral index for the initial perturbations $n=1$. Denoting with $\Omega_{i}$ the ratio between density of the generic component $i$ and the critical density to close the Universe, in our Standard CDM models we 
adopt $\Omega_{\mathrm{b}}=0.1$ (for baryons) and $\Omega_{\mathrm{c}}=0.9$ (for Dark Matter). The Hubble constant is taken to be $50 \mathrm{~km} \mathrm{~s}^{-1} \mathrm{Mpc}^{-1}$.

Little is said in literature about the choice of the initial temperature to be assigned to baryonic particles. In general, the choice depends on the temperature coverage of the cooling processes under consideration, and very often the initial temperature is set at the lowest value of the temperature interval. This is less of a problem with our code because we have implemented the large tabulations supplied by Chiosi et al. (1998) which extend down to molecular processes at low temperatures (as low as about $10-100 \mathrm{~K}$ ).

A rough estimate of the mean background temperature of the Universe at the redshift we are interested is derived as follows. At the decoupling redshift $z \sim 1000$, the temperature is about $3000 \mathrm{~K}$. After decoupling, the Thomson scattering ceases, but a sufficiently large fraction of free electrons remains to keep matter in equilibrium with radiation via Compton scattering until $z \sim 160$ (Madau 2003). Since then the temperature ever decreases (as far as matter component are concerned) proportionally to $(1+z)^{2}$. Given that we are considering over-dense regions, we assume the initial temperature of the sphere to be equal to the mean temperature of the Universe, at the time in which the mean background density was equal to the mean density of our protogalactic sphere. With the initial red-shifts we have adopted (in the range $50<z<100$ ), the temperature falls in the interval 10 to $150 \mathrm{~K}$.

\subsection{Final parameters for the galaxy models}

For all simulations the initial smoothing length parameter for the baryonic component has been set equal to ten times the softening length. The softening length $\epsilon$ in turn has been chosen in such away that both a good resolution and the Evrard (1988) relation are secured. This latter requires

$\frac{G m}{\epsilon} \ll \frac{G M}{R}$

i.e. $\epsilon \gg R / N_{\text {part }}$. In Table 2 are listed the dynamical and computational parameter we have adopted for the three models (the cosmological parameters have already been given in Table 1).

The basic units for length, time ad mass used to express all physical quantities entering the models are:

- length: 1 proper Mpc;

- time: the lifetime of the Universe, corresponding to the cosmological model adopted for the simulation;

- mass: the unitary mass is obtained supposing the free-fall time-scale of a sphere with unitary radius equal to the unit time so that the gravitational constant $G=1$ in code units.

\section{Galaxy models: the results}

Model A has been evolved up to age of about 13 Gyr (i.e. the age of the Universe), whereas model B has been followed up to 5 Gyr. Nevertheless, the last computed model is well beyond the stage of maximum stellar activity, i.e. the galaxies have already settled down to a passive evolutionary regime.

\subsection{Spatial evolution}

The series of Figs. 1 through 6 show the positions of Dark Matter, gas and star particles projected on the $x y$ plane at different values of the redshift for models A and B as indicated. It
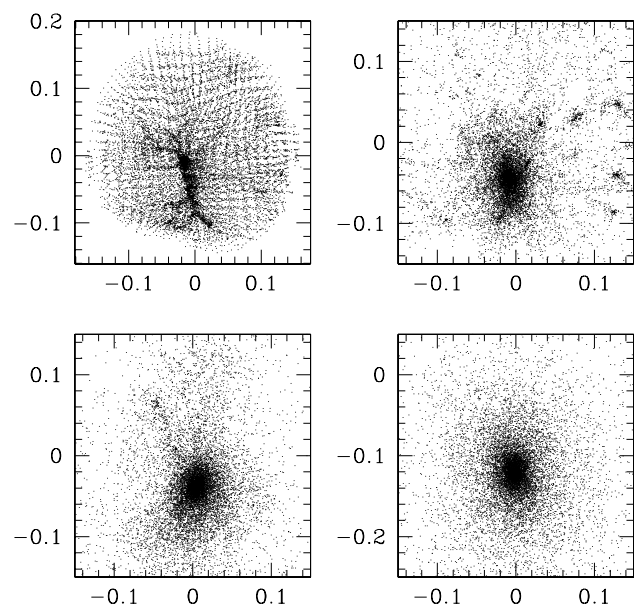

Fig. 1. Positions on the $x y$ plane of the Dark Matter particles for model A (proper Mpc) at different times; from left to right and top to bottom, $z=7.6,3.2,1.6,0$.
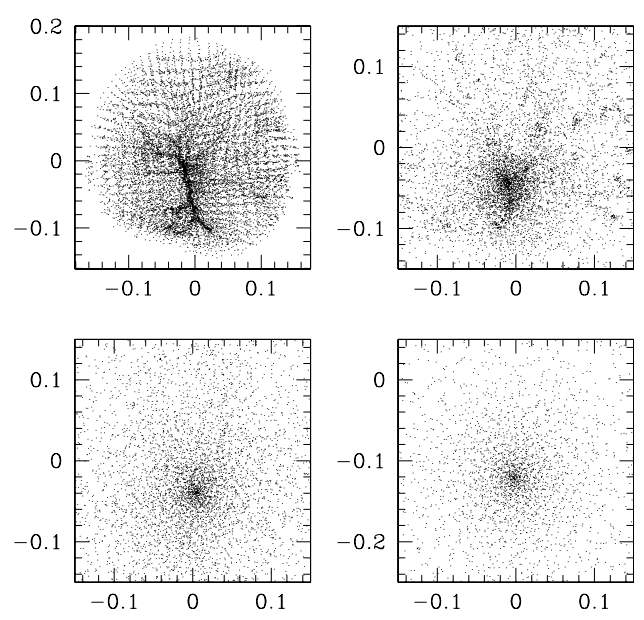

Fig. 2. The same as in Fig. 1 but for the gas particles.
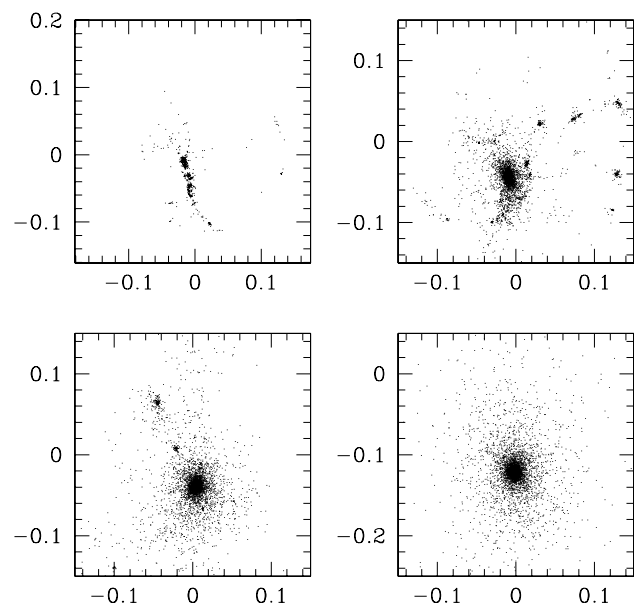

Fig. 3. The same as in Fig. 1 but for the star particles.

must be noticed that the panels for model A has length scale of $300 \mathrm{kpc}$ along both axes, whereas the length is $100 \mathrm{kpc}$ in the case of model B.

One may sketch the following evolutionary picture for the two galaxies. The proto-galaxy initially expands with the Hubble flow and the expansion becomes slower and slower until it detaches from it first in the central regions (or in general in the 

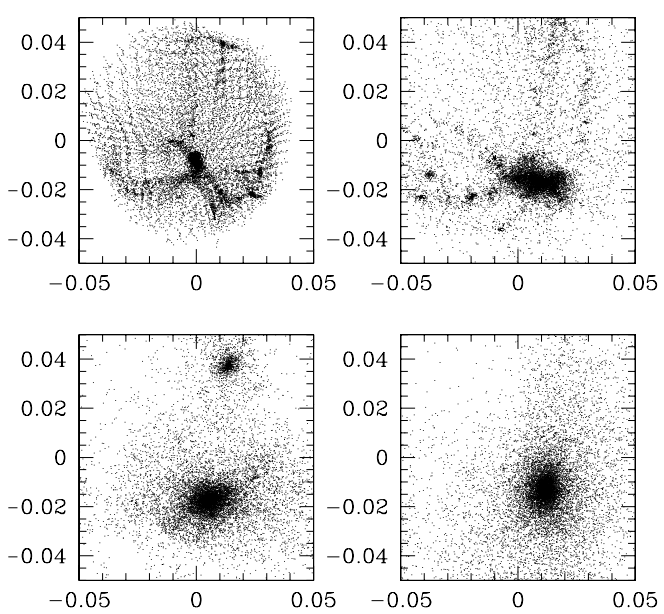

Fig. 4. Position on the $x y$ plane of the Dark Matter particles for model B (proper Mpc) at different times; from left to right and top to bottom, $z=6.3,4.2,2.2,1.0$.
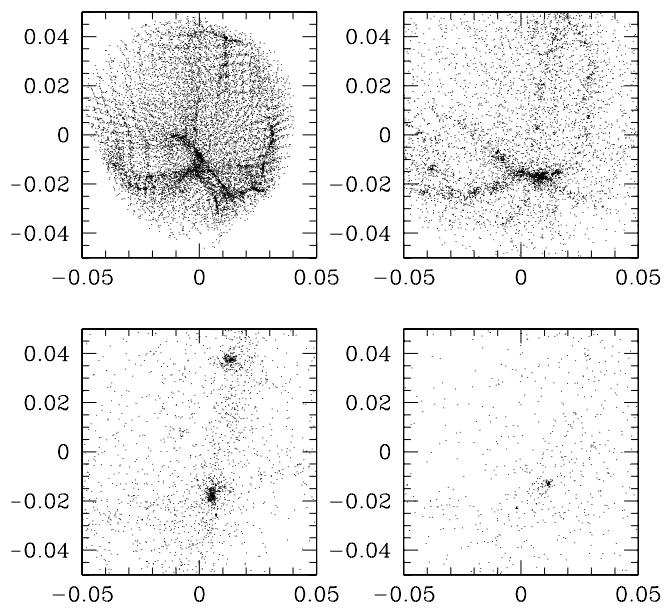

Fig. 5. The same as in Fig.4 but for the gas particles.

denser regions) and later in the outskirts. Not all gas is able to collapse but a certain (often huge) fraction remains at the edges of the just born galaxy. This is the result of the inhomogeneous distribution of the initial densities that lead some regions to collapse earlier than others independently of their distance from the centre of the system. As a whole, the collapse deviates from the ideal spherical case. In particular, one may stress that the final baryonic-to-Dark ratio will be different from the initial one, in the virialized region of the galaxy. In model A the density peak is nearly uniformly distributed around the geometrical centre; it forms a massive peak and several small sub-units. In model B we note the evolution of two distinct units, one evidently smaller than the other, which eventually merge to form a single object; the merger is completed at $z \sim 1.3$.

Once the collapse has started, while the collisionless Dark Matter begins to form clumps of high density the gas is decelerated and heated up by its own viscous friction which causes the onset of shock processes; the kinetic energy of the gas is largely turned into internal energy by such shocks; strong cooling begins to occur, thus initiating star formation. Baryonic matter, in the form of stars, can now freely fall in the potential wells of Dark Matter.

The first stars, in general in small isolated groups, start to form at $z \simeq 12$ in model $\mathrm{A}$ and $z \simeq 15$ in model B. Star formation proceeds and as shown below it occurs in a sort of broad burst
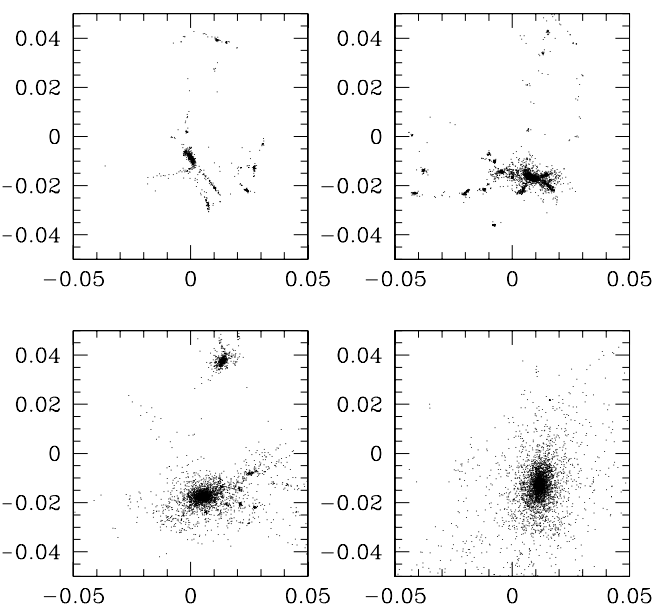

Fig. 6. The same as in Fig. 4 but for the star particles.

whose duration is of the order of one or two Gyrs; then it slows down until it (almost) stops.

Following the overall collapse, the energy injection by shocks and stellar feedback increases the energy (temperature) of the gas left over by stars to the point that part of it can overwhelm the gravitational potential. The gas escapes the galaxy and star formation is eventually quenched off.

At the end of the galaxy and star formation period, the morphology of all our models seems to nicely resemble that of real galaxies.

To highlight the structure of our model galaxies, first we analyse their three-dimensional shape. To this aim we chose a suitable cut-off radius and consider the stellar and Dark components inside this radius. The axial ratios can be roughly estimated as

$\frac{b}{a}=\sqrt{\frac{\Sigma m_{i} y_{i}^{2}}{\Sigma m_{i} x_{i}^{2}}} \quad \frac{c}{a}=\sqrt{\frac{\Sigma m_{i} z_{i}^{2}}{\Sigma m_{i} x_{i}^{2}}}$

where $x, y, z$ are the coordinates along the axis of the resulting ellipsoid. For model A, inside the half mass radius, we find the axial ratios $b / a=1.08$ (stars) and 1.14 (Dark Matter), and $c / a=1.07$ (stars) and 1.17 (Dark Matter). For model B we get $b / a=1.04$ (stars) and 1.14 (Dark Matter), and $c / a=1.00$ (stars) and 0.96 (Dark Matter). We can therefore state that, in general, the stars are nearly spherically distributed, while the Dark Matter haloes show a weak asymmetry. Owing to the smallness of the deviation, the two models are considered as spherically symmetric.

The panels of Fig. 7 show the final surface density profiles of the stellar contents of models A and B projected onto the $x y$ plane when observed face on. The solid line are the Sersic (1968) surface density profiles

$\Sigma=\Sigma_{\mathrm{e}} \exp \left\{-(0.324-2 m)\left[\left(R / R_{\mathrm{e} B}\right)^{1 / m}-1\right]\right\}$

where $R_{\mathrm{e} B}$, the effective radius, is the radius containing half mass of the galaxy (in this case referred to cylindrical symmetry), $\Sigma_{\mathrm{e}}$ is the mass density inside this radius, and $m$ is a numerical parameter which correlates with the galaxy absolute magnitude; for $m=1$ one finds the exponential law of dwarf ellipticals, while for $m=4$ one gets the de Vaucouleurs (1948) law. The agreement between the numerical results and the de Vaucouleurs law is remarkably good for model A; only in the very central regions (i.e. $r \leq 2 \mathrm{kpc}$ ) the density of star particles seems to be too peaked. Model B seems well fitted by a Sersic law with $m \sim 6$. 

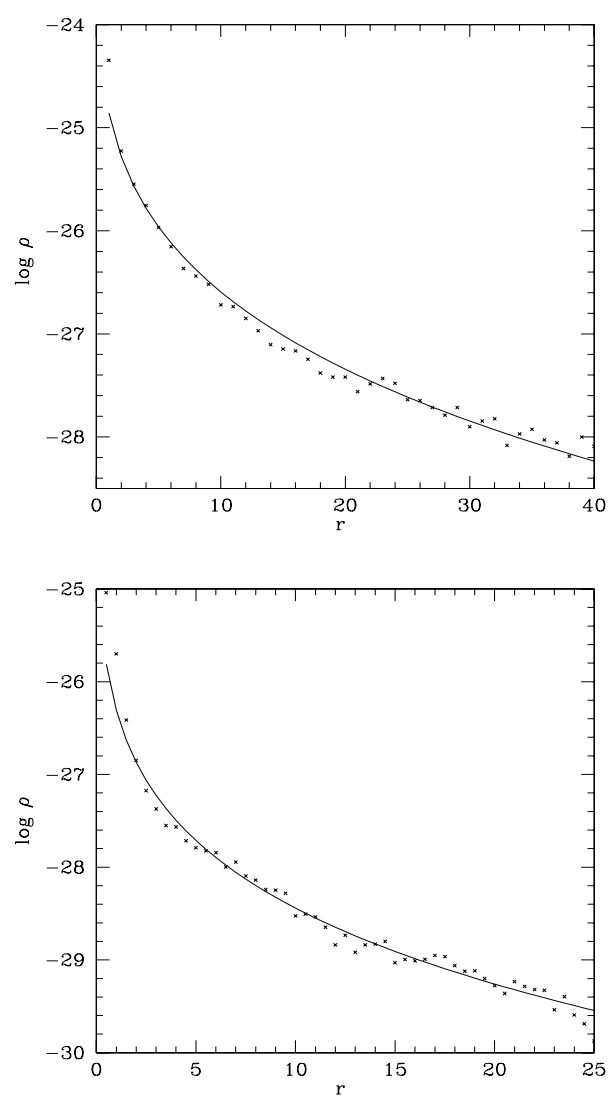

Fig. 7. Top panel: final surface density profile for model A $\left(\log \left[\mathrm{g} / \mathrm{cm}^{2}\right]\right.$ vs. kpc). The dots are averaged surface densities projected on the $x y$ plane. The solid line is a Sersic law (see text for details). Bottom panel: the same but for model B.

The best-fits have been made minimizing the dispersion of the points $\sqrt{\left[\rho_{i}(r)-\rho_{\text {fit }}(r)\right]^{2}}$ with respect to the analytical relationship. As noticed by Caon et al. (1993), one would actually expect smaller values of $m$ for smaller galaxies, while we obtain the opposite. This is a drawback of the present models, which however should not be rejected because of it. Profiles with $m=6$ and $m=4$ are indeed quite similar, so that most likely the present simulations do not possess the resolving power to distinguish between the two. It is safe to say that the general trends of the mass profiles resemble those of real galaxies, but a detailed comparison between theory and observations is not yet possible with the present models.

Considering the models as roughly spherical, we try to fit their three-dimensional density profiles with theoretical analytical laws, i.e. the Hernquist (1990) law for the stellar component

$\rho(r)=\frac{M}{2 \pi} \frac{a}{r} \frac{1}{(r+a)^{3}}$

where $M$ is the total mass of the stellar system and $a$ is a scale length that depends on the half-mass radius $r_{1 / 2}=(1+\sqrt{2}) a$, and the Navarro et al. (1996) universal profile for the Dark Matter component

$\frac{\rho(r)}{\rho_{\text {crit }}}=\frac{\delta_{\mathrm{c}}}{\left(r / r_{\mathrm{s}}\right)\left(1+r / r_{\mathrm{s}}\right)^{2}}$

where $\rho_{\text {crit }}$ is the critical density, $r_{\mathrm{s}}$ is a scale radius at which the slope of the density profile (33) is -2 , and $\delta_{\mathrm{c}}$ is a characteristic (dimensionless) density that depend on the cosmological model and $r_{\mathrm{s}}$ (see Navarro et al. 1996, for details).
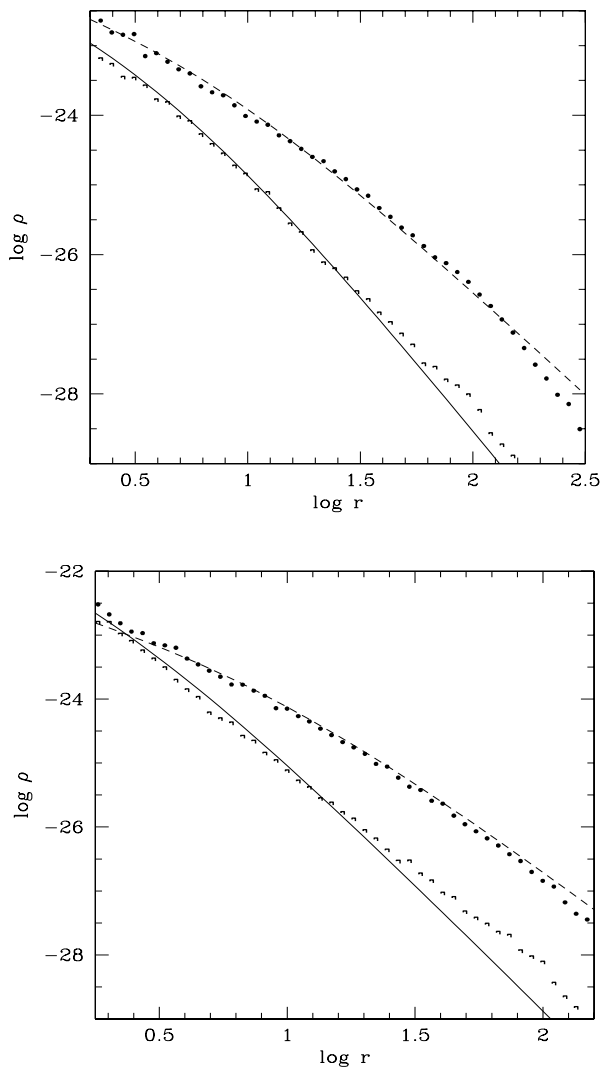

Fig. 8. Top panel: final spherical density profile for model A $\left(\log \left[\mathrm{g} / \mathrm{cm}^{3}\right]\right.$ vs. $\left.\log [\mathrm{kpc}]\right)$. Dots and squares are respectively CDM and stars averaged densities in spherical shells at the corresponding radius. The solid line is the Hernquist law; the dotted line is the NFW profile. Bottom panel: the same but for model B.

Figure 8 shows the mass densities for our stellar and Dark Matter components compared with the theoretical profiles (fits are obtained the same way as above): the agreement seems quite good in the middle regions. Obviously, little can be said about the most central regions (i.e. less than $\sim 2.5 \mathrm{kpc}$ ), due to the effects of the softening length and, in general, of numerical nature (see e.g. Power et al. 2003, for a discussion of these issues).

For both models the Dark Matter profile is very steep in the innermost central regions. Current cosmological simulations give profiles with inner slopes between $\sim-1$ (Navarro et al. 1996) and $\sim-1.4$ (Moore et al. 1998). However, there are evidences that the inclusion of non-adiabatic treatment of the gaseous component, and particulary the star formation process, could steepen the Dark Matter density profile (e.g. Lewis et al. 2000; Kawata \& Gibson 2003; Gnedin et al. 2004). Power et al. (2003) also argue that poor temporal resolutions could give rise to artificial cusps in the innermost regions. Therefore, considering the uncertainty still affecting the whole issue, the present results are acceptable.

Furthermore, we note that, contrary to common expectations, in the central regions of our models the density of Dark Matter exceeds that of baryonic matter (stars): the Dark Matter halo seems to be strongly concentrated. We adopt the definition of concentration by Navarro et al. (1996) as the ratio $c=R_{\mathrm{vir}} / r_{\mathrm{s}}$ between the virial radius $R_{\mathrm{vir}}$ of the Dark Matter halo and the scale radius of relation (33) $r_{\mathrm{s}}$. As virial radius we adopt the radial distance at which the mean total density of the systems is 200 times the background density; so, in this estimate both Dark and Baryonic matter are included. However, owing to the small 
Table 3. Masses and half mass radii for the various components of models A and B. Masses are in units of $10^{12} M_{\odot}$, radii are in kpc.

\begin{tabular}{lll}
\hline \hline Model & $\mathrm{A}$ & $\mathrm{B}$ \\
\hline Final star mass $M_{\mathrm{s}}$ & 0.091 & 0.0029 \\
Final gas mass (collapsed) & 0.062 & 0.0004 \\
$M_{\mathrm{s}} / M_{\text {bar }}$ & 0.56 & 0.82 \\
Half mass radius of stars & 7 & 1 \\
Effective radius of stars & 5.2 & 0.8 \\
Sersic law index $m$ & 4 & 6 \\
Half mass radius of DM & 52 & 15 \\
Concentration (NFW profile) & 50 & 20 \\
Virial radius & 300 & 41 \\
Age of the last model (Gyr) & 13 & 5 \\
\hline
\end{tabular}

fraction of baryons with respect to the total mass, the results does not significantly depend on their presence. The scale radius is derived from the best-fit of our numerical results to the NFW profile (see Fig. 8). The concentrations we find for models A and $\mathrm{B}$ are $\sim 50$ and $\sim 20$, respectively.

These values are higher than current expectations which indicate concentration of $\sim 10$ for galactic haloes (Navarro et al. 1996). This drawback of our models requires a careful future analysis. First of all, as already mentioned, the presence of stars can steepen the Dark Matter profiles. In this contest the effects of different star formation recipes and/or different choices for the model parameters should be investigated. Second, quasicosmological initial conditions ignore the possible late-time infall of gaseous matter from the IGM into the virialized halo of the new born galaxy. Moreover, the observational estimate of the mass profiles in the centre of galaxies is derived with the aid of (theoretical) mass-to-light ratio for the stellar components, which depend on the IMF as well as stellar evolution properties. While these latter are somewhat established, this is not the case of the IMF. Passing from one IMF to another may easily change the mass-to light ratios by a large factor (Portinari et al. 2004). In addition to this, our galaxies seem to be completely relaxed and in equilibrium (see below). This probably means that a baryondominated centre is not strictly necessary to secure the stability of the systems. Last, the density profiles of the luminous component of our models fairly agree with those of Hernquist (1990). Therefore, as far as we can tell, neither theoretical nor observational evidences actually exist constraining the central regions of galaxies to be always baryon-dominated, each galaxy being peculiar in its formation and virialization history. In conclusion, we feel confident to consider our models as reliable representations of the gross scale features of real galaxies and the grand design of galaxy formation in which these numerical simulations are framed.

Finally, the effective radius of the galaxy is calculated using the Hernquist density profile, so that

$R_{\mathrm{e} B} \simeq \frac{R_{1 / 2}}{1.33}$,

(Hernquist 1990). Table 3 gives the values of the parameters concerning the global properties of the density profiles of the models.

\subsection{The star formation history}

The history of the star formation rate, in solar masses per year, is shown in Fig. 9 for model A (top panel) and B (bottom panel). They can be compared, for example, with those by Chiosi \& Carraro (2002) and Kawata (1999, 2001a,b). It is evident that
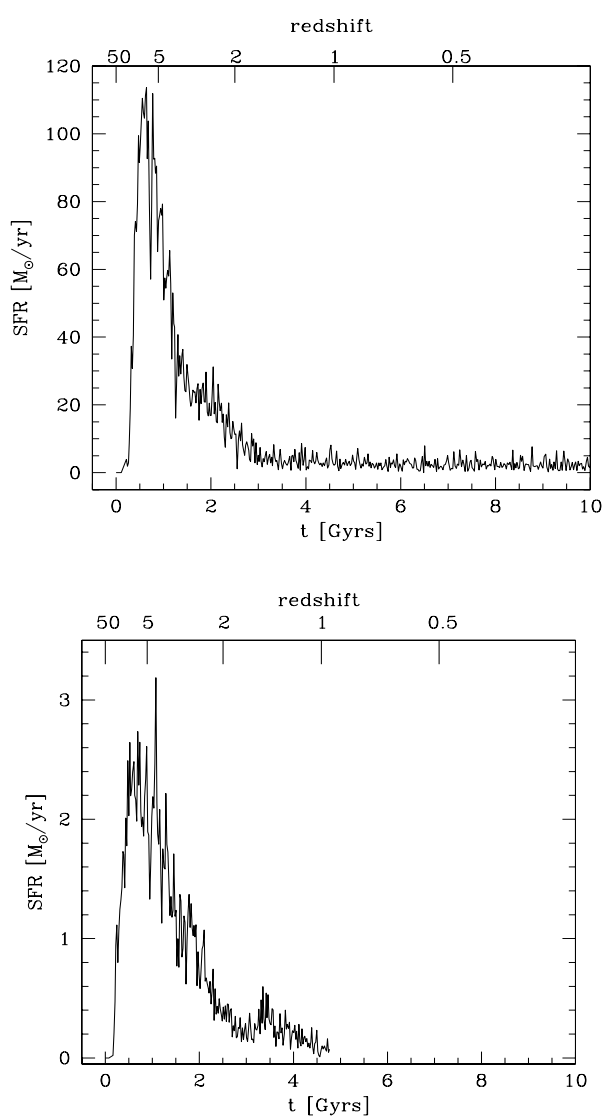

Fig. 9. Top panel: star formation rate $\left(M_{\odot}\right.$ per year $)$ in model A. Bottom panel: the same but for model B.

in all cases the galaxy goes through a phase of strong stellar activity, in a sort of burst-like mode lasting about $2 \mathrm{Gyr}$, followed by very slow activity (of the order of $1-5 M_{\odot} / y r$ ) that continues for long periods of time. Remarkably the bulk of stars are in place at the galaxy age of $2.5 \mathrm{Gyr}$, i.e. at the redshift $z=1.95$ for model $\mathrm{A}$ and $z=2.2$ for model $\mathrm{B}$.

This behaviour shares the properties of the hierarchical and monolithic scheme. It is hierarchical because in the proto-galaxy lumps of matter merge together to assemble the galaxy we see today. The merging history is complete at redshift $z=2.5$ for the model $\mathrm{A}$ and $z=2.2$ for model $\mathrm{B}$, except one major merger which happens at $z \sim 1.3$. It is monolithic because the largest fraction of the star mass is built up very early before $z=2$, for all models.

The results we have obtained clearly favour the monolithic scheme of galaxy formation (cf. Sect. 1) as a unique episode of star formation during very early stages can generate a structure very similar to what we see today; even in the case of a subsequent major merger as that of model B, the star formation history does not undergo substantial changes, perhaps due to the small amount of gas left over by the first burst of activity.

How star formation actually works obviously depends on the initial conditions. In our models, the results favour the mergers of small sub-units in very early epochs rather than the formation of big galaxies from mergers of other galaxies at much later times.

A huge fraction of the collapsed gas is turned into stars and also a considerable amount of gaseous material is blown away before it can collapse in the proto-galaxy potential well). With the present recipes, the efficiency of gas restitution by dying stars refuels the interstellar medium with sufficient new gas to 
engender minimal star formation after the galaxy has reached fully relaxed conditions. This is less of a problem here because the poor mass-resolution makes the effects on the star formation rate of the transformation of one or two particles per step more dramatic than they actually are. Moreover, slightly different conditions on the recipe for the star formation rate could get rid of it. In relation to this, many experiments have recently been made to evaluate and simulate the effects of stellar energetic feedback on the interstellar medium (cf. for instance Springel \& Hernquist 2003; Marri \& White 2003). Sufficiently strong feedback would stop the star formation activity by evaporating the cold gas clouds in which new stars would form. However the poor resolution of the present numerical simulations does not allow us to properly investigate this problem. As already pointed out, we are not able to reproduce the effects of stellar re-heating on the kinetics of gas particles, so that we must limit ourselves to just fuel the thermal budget, which actually proves to be ineffective, due to the strong cooling phenomena which dominate the gaseous non-adiabatic processes at the temperatures we are dealing with. Therefore, past the strong, dominant episode of star formation, the long tail of stellar activity can be simply ignored for all practical purposes, for instance the derivation of spectro-photometric properties.

As already mentioned, the by far dominant episode of star formation occurred at very early epochs and lasted for a significant fraction of the Hubble time. The duration of it (about 2 Gyr) is longer in model B than in model A thus leading to a higher ratio between the total baryonic mass and the left-over gas mass (cf. Table 3). All this bears very much on the chemical evolution of the (model) galaxies as discussed in Sect. 5.4.

Table 3 lists the mass of the various components at the end of the simulations together with the half mass radius of the stellar and Dark Matter distributions. CDM turns out to be strongly concentrated, as already noticed. The final stellar-to-gas ratios (considering only the collapsed and/or reprocessed gas) turns out to be $\sim 1.47$ and $\sim 7.25$ for models $\mathrm{A}$ and $\mathrm{B}$, respectively; as already pointed out, model $\mathrm{B}$ results much more efficient in forming stars, leaving only $\sim 13 \%$ of the baryonic collapsed particles in the gaseous form (see Table 3 for details). The mass of model B is not small enough to consider it a dwarf elliptical, but this results is still a bit surprising, since we would expect that a less deep potential well would produce less efficient star formation processes (see e.g. Chiosi \& Carraro 2002). Remarkably, the vast majority of stars in model B are formed before the major merger at $z \sim 1.3$, thus ruling out the possibility that the merger could be responsible for the different efficiency in forming stars (indeed, a small increase in star formation can be noticed in coincidence with the merger, but after the formation of the bulk stellar content stars). A possible explanation can be sought in the presence of two distinct potential wells. The central regions of an even small potential well can indeed be more efficient in forming stars, than the peripheral regions of a bigger one. In other words, while the gaseous particles in the outskirts of a single, deep potential well begin to heat up by stellar winds and supernova explosions before they can fall inside the well and therefore are likely subtracted from the star forming activity, in presence of two (or more) potential wells a larger percentage of gas particles, in different regions of the forming galaxy, can be turned into stellar particles.

Figure 10 shows the age of the stars as a function of their radial position for models $\mathrm{A}$ and $\mathrm{B}$. It clearly illustrates how a galaxy is assembled. The star formation process in the central regions goes on for very long periods of time; younger and more metal-rich stars are therefore expected to be found in the core of
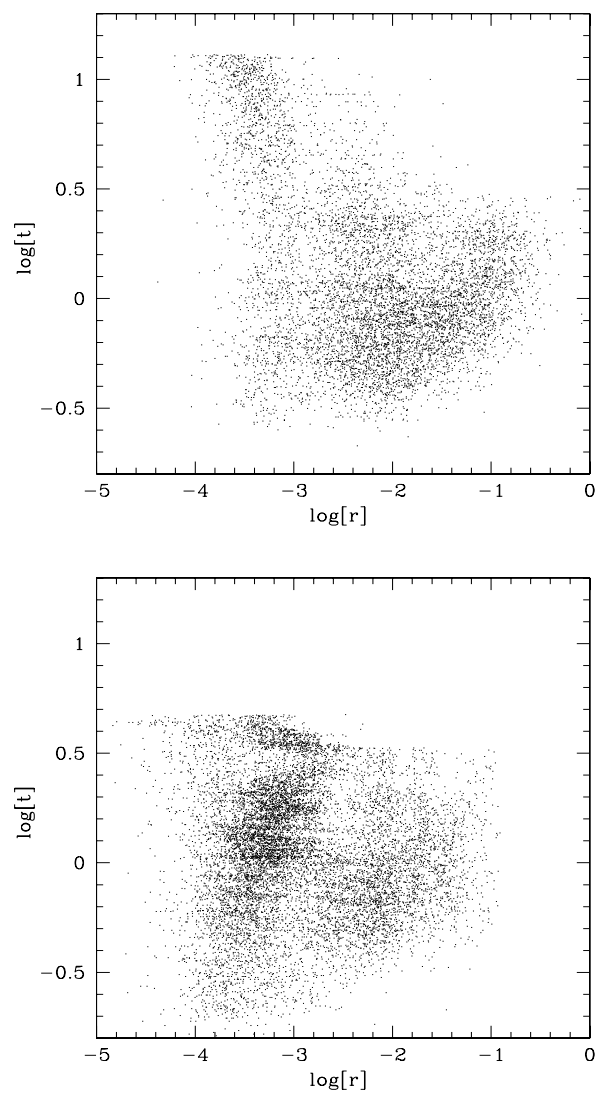

Fig. 10. Top panel: formation time $(\log [\mathrm{Gyr}])$ vs. radial distance $(\log [\mathrm{Mpc}])$ of the stars in model A. It is easy to notice how the galaxy we see today is gradually built up moving from the external regions toward the centre where star formation lasts very long. Bottom panel: the same but for model B.

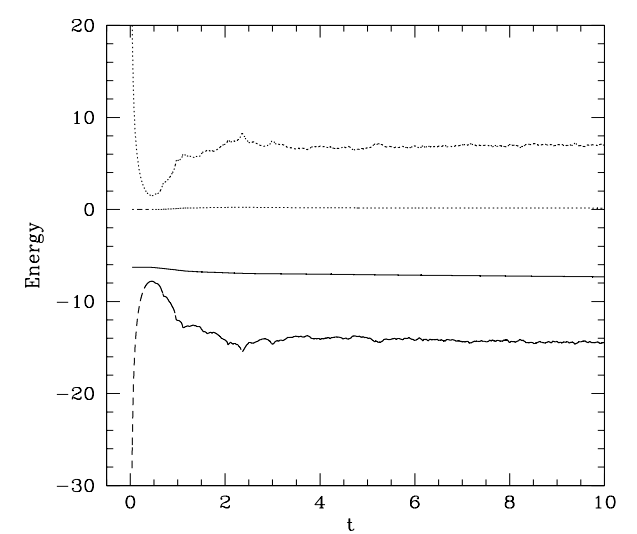

Fig. 11. Variation of (from top to bottom) kinetic, thermal, total and potential energy in code units as function of time in Gyrs for model A (all components).

galaxies, whereas older and more metal-poor stars are likely to be found in the outer regions.

\subsection{Energy}

The panels of Fig. 11, from top to bottom, show the variation as a function of time of the kinetic, thermal, total and potential energies (all expressed in the physical units we have adopted in the code) of model A. The same quantities for model B have similar behaviour. The trends resemble nicely what we expect to see in an expansion and subsequent collapse of an isolated 

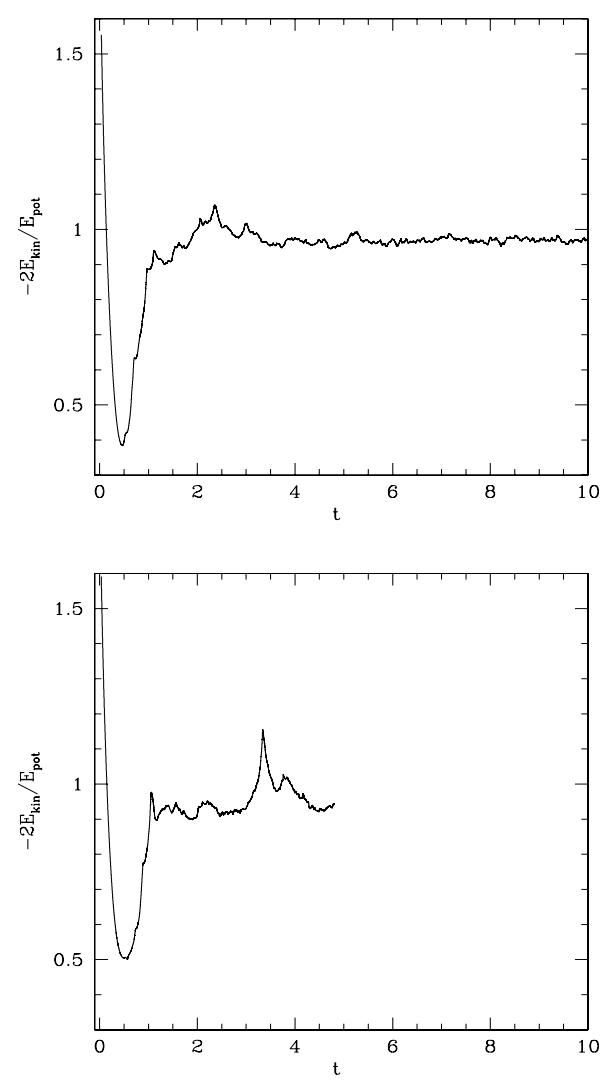

Fig. 12. Top panel: trend of the Virial trace $-2 E_{\text {kin }} / E_{\text {pot }}$ for models A and B from top to bottom. Ages are in Gyr.

system; notice that the thermal energy, which is provided only by the gas, is very small when compared to the kinetic and the potential terms. The total energy is not strictly conserved (only a few per cent), as the inclusion of non-adiabatic processes such as cooling causes the loss of part of the energetic budget of the gaseous component.

Figure 12 shows the Virial Trace $V=-2 E_{\text {Kin }} / E_{\text {pot }}$ as a function of time for the two models. It is easy to follow the relaxation of the system, which tends to a state of equilibrium after some small oscillations (if the systems had been strictly collisionless and more symmetrical these oscillation would have been much stronger, as we expect in a violent relaxation process). The expected value for an ideal system would be $V=1$, but here the presence of viscosity and cooling of the gas tend to shift the ratio to somewhat lower values to the loss of energy. It is worth noticing in model $\mathrm{B}$ the bump in coincidence with the major merger occurring at $\sim 3.5 \mathrm{Gyr}$.

Moving now to heating processes, the rate of supernova explosions of model A is shown in Figs. 13 (the trend is nearly the same for model B). The top and bottom panels are for type IA and type II supernovae, respectively. As expected the rate of type II supernovae strictly follows the star formation rate: as massive stars are short lived, as soon as star formation starts and/or ceases the same does the type II supernova rate. The case of type Ia is different because they require a certain amount of time to show up and continue to explode for very long time after the end of the star forming period.

\subsection{Metallicity}

Figure 14 displays the evolution of the mean metallicity $Z$ and mass abundance of $\mathrm{Fe}$ and $\mathrm{O}$ both for stars and gas in models $\mathrm{A}$
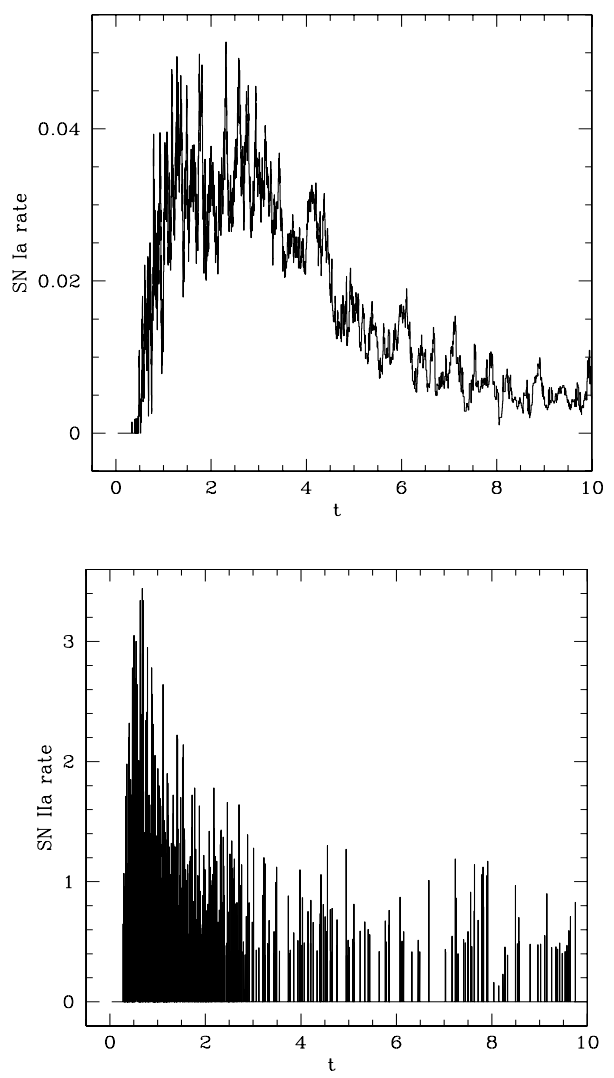

Fig. 13. Top panel: type Ia supernova rate (explosions per year) as function of time for model A. Bottom panel: the same as above but for type II supernovae.

and $\mathrm{B}$; the ratio $[\mathrm{O} / \mathrm{Fe}]$ is in the standard spectroscopic notation. The plots can be compared with those in Fig. 5 of Chiosi \& Carraro (2002).

Looking at the $[\mathrm{O} / \mathrm{Fe}]$ vs. age relation displayed in Fig. 14, we notice that the so-called $\alpha$-enhancement problem is present. Both models have the stellar content strongly enhanced in $\alpha$-elements, however the degree of enhancement is lower in model B (lower total mass) and in both cases decreases with the age. These trends are agree with the observational information, see the review by Chiosi (2000), and the similar analysis made by Tantalo \& Chiosi (2002) using the Chiosi \& Carraro (2002) models.

The final mean stellar metallicity in model A is nearly solar $\left(\langle Z\rangle \simeq 0.8 Z_{\odot}\right)$. Remarkably also model B shows similar values of mean metallicity even if its evolution was stopped much before the present age, thus showing how the chemical enrichment seems to be almost completed in the first Gyr of a galaxy life.

Figure 15 shows the final number distribution of stars per metallicity bin $\left(Z / Z_{\odot}\right)$, limited to model $\mathrm{A}: 4 \%$ of stars have mean total metallicity lower than $1 / 3$ solar, $67 \%$ have mean total metallicity in the range $1 / 3$ solar to solar, a significant fraction goes up to three times solar, and a tiny fraction even up to 5 times solar. The low fraction with metallicity lower than $1 / 3$ solar secures that the so-called G-dwarf problem does not occur, whereas the tiny fraction around 5 times solar could explain the UV excess in elliptical galaxies. See Bressan et al. (1994) for a thorough discussion of these topics.

At any age a large scatter in the metallicities of the star particles has to be expected. This is shown in Fig. 16. The situation resembles the one observed among the stars of the Galactic Disk (Edvardsson et al. 1993b,a). 

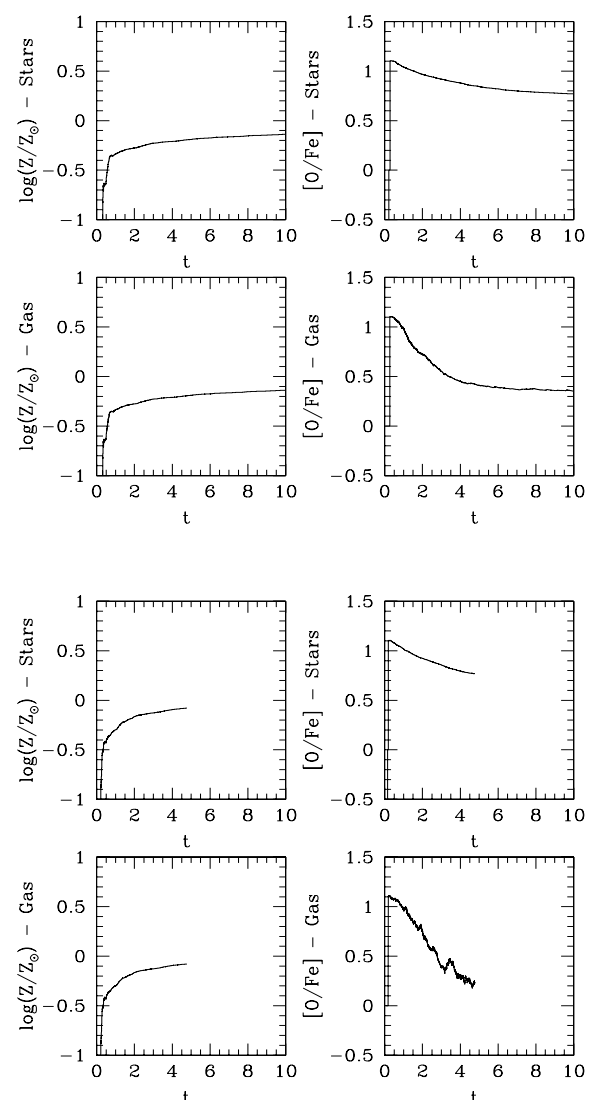

Fig. 14. Top panel: chemical evolution of the star and gas particles in model $\mathrm{A}$ as a function of the age; left panels show the mean total metallicity $\mathrm{Z}$ in solar units, right panels the ratio $[\mathrm{O} / \mathrm{Fe}]$. The age is in Gyrs. Bottom panel: the same as above but for model B.

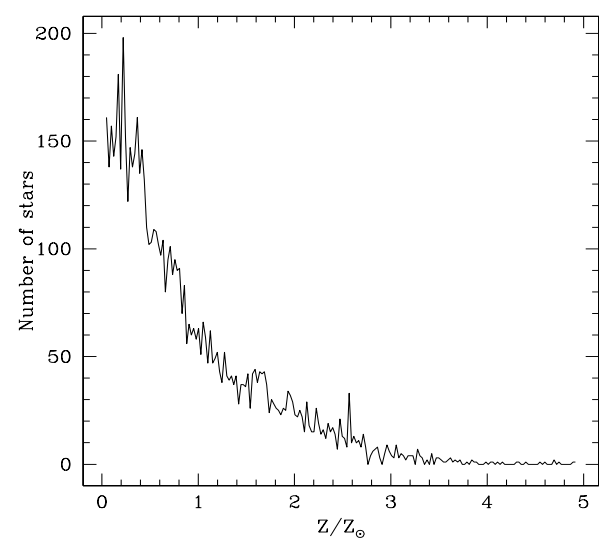

Fig. 15. Number distribution of stars in metallicity at the end of the simulation for model A.

Finally, in Fig. 17 we display the spherically averaged radial gradient in metallicity for model A and B; stars closer to the centre are also more metal rich. For elliptical galaxies, Davies et al. (1993) find the metallicity gradient $\Delta \log (Z) / \Delta \log (r) \simeq$ $-0.2 \pm 0.1$. The gradient of model $\mathrm{A}$ is $\simeq-0.30$, in good agreement with the observational estimate; model B shows instead a steeper gradient, $\simeq-0.50$, due to the larger amount of stars produced towards the centre.

As a last remark we like to report here that similar simulations with almost identical initial conditions but with the Arimoto \& Yoshii (1987) IMF yield very high values of the
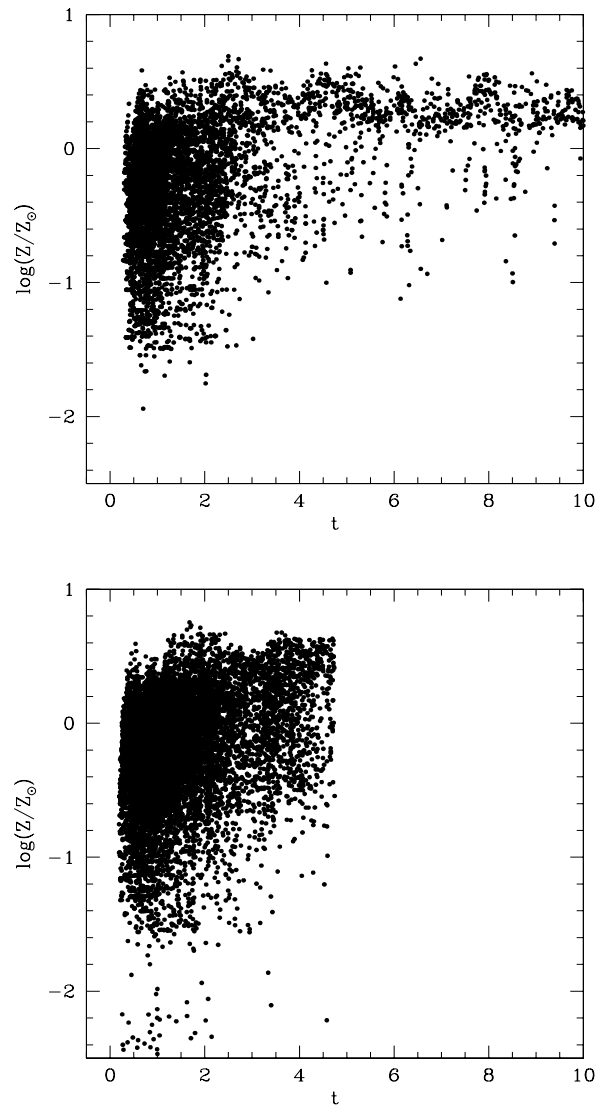

Fig. 16. Top panel: metallicity $\left(\log \left[Z / Z_{\odot}\right]\right)$ vs. age of formation (Gyrs) of the stars in model A. Bottom panel: the same but for model B.
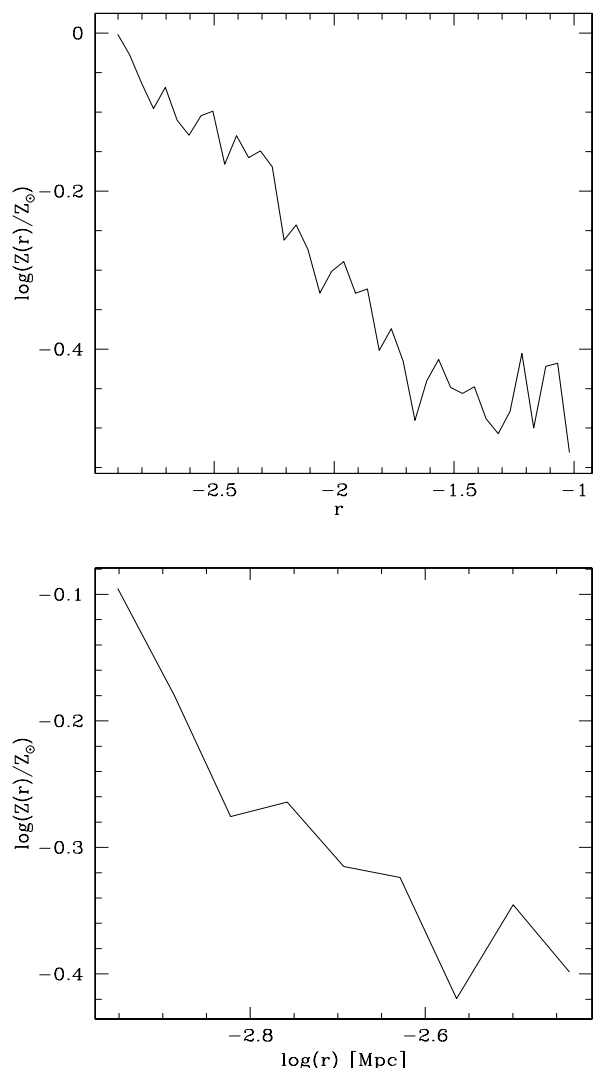

Fig. 17. Radial gradient in the spherically averaged metallicity for the stars of model A (top) and B (bottom). Radii are in (log[Mpc]). 
final mean metallicities, i.e. up to 5 or 6 times the solar abundance (Merlin 2005). The reason is that the Arimoto \& Yoshii (1987) IMF favour massive stars and type II supernovae and therefore predict much higher rates of gas restitution than the Kroupa (1998) or Salpeter (1955) IMF (see e.g. Lia et al. 2002). As such high mean metallicities do not agree with current observational estimates, in the present study we prefer to abandon the Arimoto \& Yoshii (1987) IMF even if it was originally tailored to model elliptical galaxies. The recent calculation of stellar yields by Portinari et al. (1998) which are incorporated in the Lia et al. (2002) algorithm for chemical enrichment in $N$-Body PD-TSPH simulations, show that IMFs too skewed toward massive stars are not required. The reader should refer to Merlin (2005) for all details about these models with Arimoto \& Yoshii (1987) IMF.

\subsection{Galactic winds}

As already reported in Sect. 1 elliptical galaxies ought to suffer galactic winds in order to explain the colour-magnitude relation. To investigate this important issue, in Fig. 18 we show the temperature vs. density relationships for all gas particles of our models. The gas left over by star formation is made of four components - hot and cold, chemically enriched and unprocessed (cf. Chiosi \& Carraro 2002). The hot gas, which is the dominant component, has relatively high densities: heated up by energy injection is about leaving the potential well of the galaxy. The fact the hot gas constitutes almost the totality of the gas content is due to the efficiency of the energy feed-back and the generally large total mass of the systems (relatively deep potential wells) which inhibits the occurrence of earlier galactic winds (cf. Chiosi \& Carraro 2002; Kawata \& Gibson 2003). Note that in model B, as fewer gas particles are left over by star formation, the situation is less defined then in model A, where we can clearly see the final dependence, for the hot component, of temperature on density.

Figure 19 shows the radial velocity of the gas particles together with the radial profile of the escape velocity. The amount of gas (excluded the fraction which did not take part to the collapse of the proto-galaxy) which, at the end of our simulations, has the radial component of its velocity greater then the escape velocity at its distance from the centre of mass of the system, can freely leave the potential well of the galaxy and can be considered as gaseous mass ejected as galactic wind. In all simulations there is a layer beyond which all the gas particles meet these requirement; the percentage of the gas which can be considered as galactic wind is $\sim 21 \%$ (model A) and $\sim 58 \%$ (model B) on the total of collapsed gas. Note that these percentages are very different from one case to another; this could be due to several factors, among which the different star formation history (as already pointed out, star formation is much more efficient in model B than model A, thus leaving a smaller amount of gas in the central regions of the system), and the different masses of the systems, since galactic winds are expected to be more efficient in galaxies of low mass (Chiosi \& Carraro 2002). At large distances from the galaxies, some particles with almost zero radial velocity can be found (see Fig. 19). They are the un-collapsed gas particles that did not take part to the galaxy formation process. They cannot be considered as galactic wind as they have not been blown away from the galaxies.

Finally, we would like to note that a better treatment of the gaseous component should be considered such as the multiphase scheme of Marri \& White (e.g. 2003) that would allow us to describe in a more realistic fashion both chemical enrichment and galactic winds.
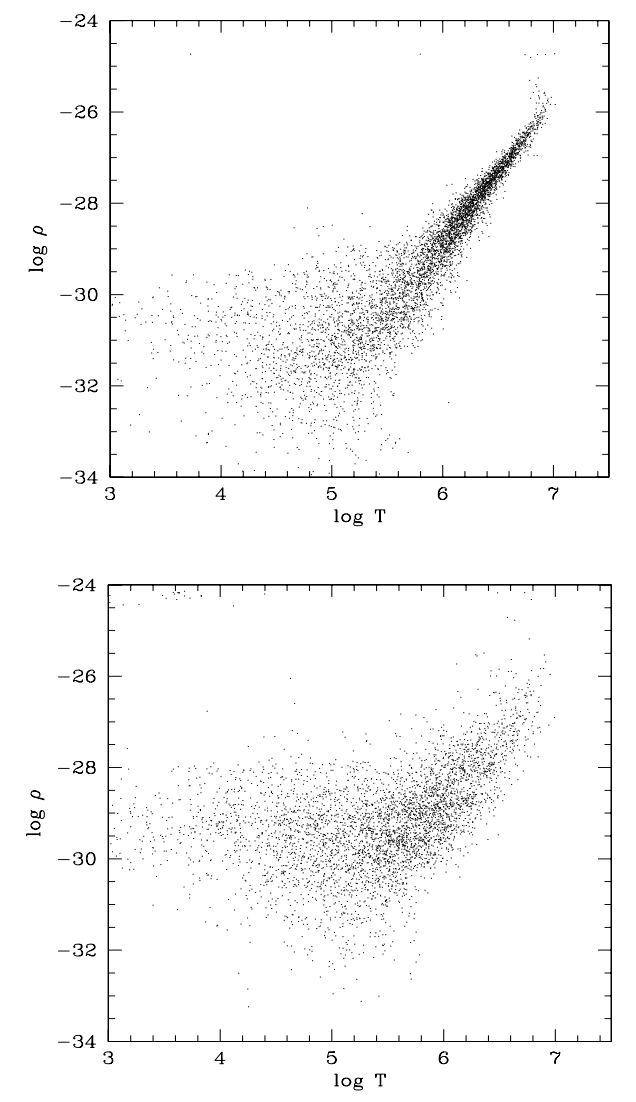

Fig. 18. Top panel: gas density $\left(\log \left[\mathrm{g} / \mathrm{cm}^{3}\right]\right)$ vs. temperature $(\log [\mathrm{K}])$ of model A. Mid panel: the same but for model B.
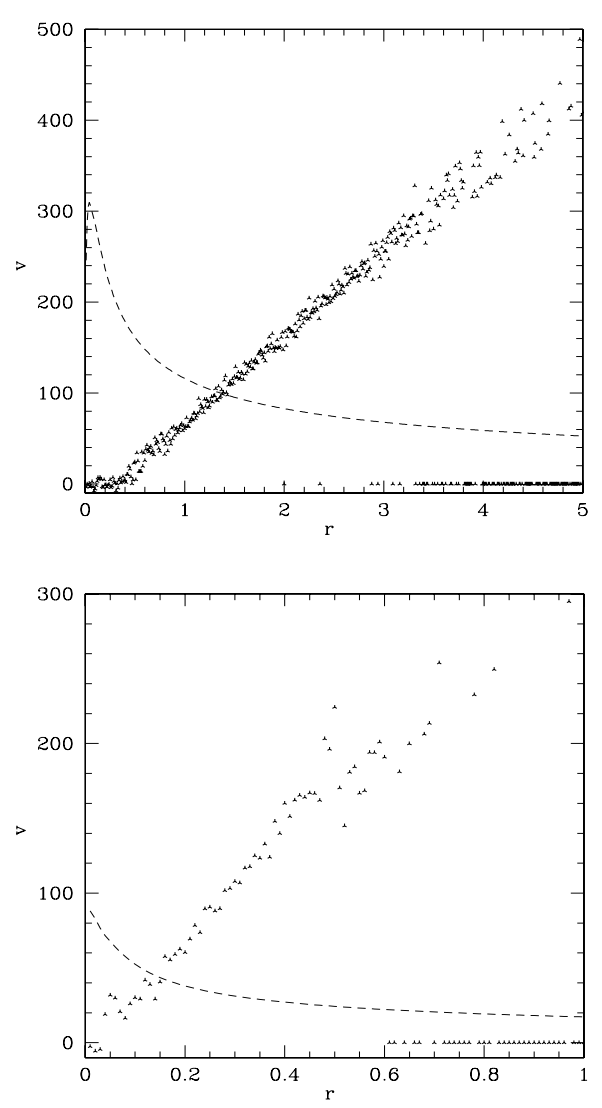

Fig. 19. Top panel: radial velocity $\left(\mathrm{km} \mathrm{s}^{-1}\right.$, points) and escape velocity (dotted line), vs. radius (Mpc) for model A. Both velocities are in proper $\mathrm{km} \mathrm{s}^{-1}$. Bottom panel: the same but for model B. 


\section{Conclusions}

In this study, with the aid of $N$-body simulations based on quasicosmological initial conditions, we have followed the formation and evolution of two early-type galaxies from the stage when they separate from global expansion of the Universe to their collapse to virialized structures, the formation of stars and subsequent nearly passive evolution up to the age of 13 Gyr. The cosmological background is the Standard CDM. We have highlighted the structural, dynamical and chemical properties of the models to be compared with observational data.

The models contain several parameters, among which we recall

- the softening parameter for Dark Matter, gas and stars;

- the specific efficiency $c_{*}$ driving the star formation rate;

- the constant $k_{\mathrm{SN}}$ defining the fraction of energy released by a supernova explosion which goes into the gas as thermal energy.

The uncertainty on these parameters opens the gate to future studies; better fine tuning of these quantities is required to get results in very close agreement with the observational data. Nevertheless the results we have obtained are satisfactory and in agreement with other studies of the same subject.

Two systems made of Dark and baryonic matter, in current cosmological proportions, with positions and velocities for both type of particles derived from the spectrum of cosmological perturbations suited to the cosmological model under consideration have been investigated.

All models started from the conform expansion of the Hubble flow, with the lowest rigid body rotation allowed by the CDM Universe (Barnes \& Efstathiou 1987). They all have been followed for a large fraction of the Hubble time, through the phases of turn around and subsequent collapse of the galactic proto-clouds of gas to small clumps caused by the initial density perturbations to the formation of equilibrium structure made of Dark Matter, stars and remaining gas. Hydrodynamical and thermal processes caused by cooling, star formation, chemical enrichment, and heating have been included and followed in detail.

The final systems morphologically resemble real elliptical galaxies of intermediate dimensions; this is proved by their surface density profiles matching the theoretical ones by de Vaucouleurs (1948) and/or Sersic (1968) that are expected for galaxies of the same mass and scale lengths. Hjorth \& Madsen (1991) have indeed showed that violent relaxation in a deep potential well leads to systems obeying the de Vaucouleurs law. Katz \& Gunn (1991) and Kawata (1999) outline that dissipative processes, followed by the PD-TSPH code in great detail, bear very much on the final results.

Star formation lasts longer in the central regions than in the outskirts thus producing gradients in metallicity that closely agree with the observational data for elliptical galaxies (Davies et al. 1993).

It is woth stressing that in any case the models still need to be improved in several aspects: (i) First the star formation that even if shaped in a huge initial burst followed by quasi-quiescence, never completely stops, because of poor mass resolution and/or still inadequate prescription for the physical conditions at which stars can be formed. The long tail of minimal stellar activity does indeed affect the spectro-photometric properties (e.g. colours) of the model galaxies that would disagree with observational data. (ii) Second, the density profiles that are not fully satisfactory, as in the central regions the baryons do not dominate the spatial density of matter as suggested by the observational data. (iii) Third, there's no right correspondence between the values of Sersic parameter $m$ to galaxies of different mass and the those derived from observational data. (iv) Finally, the predicted massmetallicity relation as compared to the one inferred from observational data (colours, e.g. the CMR). The present model B with lower mass actually suffers more star formation and metal enrichment in turn. The reason for that is not yet clear. The present results cannot yet recover the metallicity-mass relationship obtained by Chiosi \& Carraro (2002) that fairly agreed with the observational information.

Nevertheless, it is worth noticing that our main results are in good agreement with those by Kawata (1999), who used the same method to generate the initial conditions, as well as with those by Kawata \& Gibson (2003) and Kobayashi (2005), who instead used the more refined method otherwise known as resimulation technique (see Sect. 2.2). This point is particularly relevant as it shows that our approach, developed independently and in parallel to that of Kobayashi (2005), can be considered sufficiently accurate.

Our simulations support the revised monolithic scenario of galaxy formation. Through the hierarchical collapse of Dark Matter haloes, clouds of gas meet, heat up, cool and eventually collapse forming stars in a single episode of star formation occurred in the far past, during the virilization of the proto-galaxy, perhaps followed by minimal stellar activity in the central regions and almost negligible morphological and structural evolution.

Acknowledgements. The authors would like to thank Laura Portinari, Bepi Tormen, Mauro D'Onofrio and Luigi Secco for the many helpful discussions and constructive criticism, Lorenzo Piovan for carefully reading the manuscript, and the anonymous referee for the many useful suggestions. This study has been financed by the University of Padua under the special contract "Formation and evolution of elliptical galaxies: the age problem".

\section{References}

Arimoto, N., \& Yoshii, Y. 1987, A\&A, 173, 23

Barger, A. J., Cowie, L. L., Smail, I., et al. 1999, AJ, 117, 2656

Barnes, J., \& Efstathiou, G. 1987, ApJ, 319, 575

Barnes, J., \& Hut, P. 1986, Nature, 324, 446

Barnes, J. E., \& Hernquist, L. 1996, ApJ, 471, 115

Bell, E. F., Wolf, C., Meisenheimer, K., et al. 2004, ApJ, 608, 752

Bennett, C. L., Halpern, M., Hinshaw, G., et al. 2003, ApJS, 148, 1

Benz, W. 1990, in Numerical Modelling of Nonlinear Stellar Pulsations Problems and Prospects, 269

Bertschinger, E. 2001, ApJS, 137, 1

Binney, J., \& Tremaine, S. 1987, Galactic dynamics (Princeton, NJ: Princeton University Press), 747

Bouwens, R. J., Thompson, R. I., Illingworth, G. D., et al. 2004, ApJ, 616, L79

Bower, R. G., Lucey, J. R., \& Ellis, R. S. 1992, MNRAS, 254, 601

Bressan, A., Chiosi, C., \& Fagotto, F. 1994, ApJS, 94, 63

Bressan, A., Chiosi, C., \& Tantalo, R. 1996, A\&A, 311, 425

Bundy, K., Ellis, R. S., Conselice, C. J., Cooper, M., Weiner, B., Taylor, J., Willmer, C., \& DEEP2 Team. 2005, American Astronomical Society Meeting Abstracts, 207

Buonomo, F., Carraro, G., Chiosi, C., \& Lia, C. 2000, MNRAS, 312, 371

Caon, N., Capaccioli, M., \& D’Onofrio, M. 1993, MNRAS, 265, 1013

Carraro, G., Lia, C., \& Chiosi, C. 1998, MNRAS, 297, 1021

Chiosi, C. 2000, A\&A, 364, 423

Chiosi, C., \& Carraro, G. 2002, MNRAS, 335, 335

Chiosi, C., Bressan, A., Portinari, L., \& Tantalo, R. 1998, A\&A, 339, 355

Davies, R. L., Sadler, E. M., \& Peletier, R. F. 1993, MNRAS, 262, 650

de Vaucouleurs, G. 1948, Annales d'Astrophysique, 11, 247

Edvardsson, B., Andersen, J., Gustafsson, B., et al. 1993a, A\&A, 275, 101

Edvardsson, B., Andersen, J., Gustafsson, B., et al. 1993b, A\&AS, 102, 603 
Eggen, O. J., Lynden-Bell, D., \& Sandage, A. R. 1962, ApJ, 136, 748 Evrard, A. E. 1988, MNRAS, 235, 911

Gnedin, O. Y., Kravtsov, A. V., Klypin, A. A., \& Nagai, D. 2004, ApJ, 616, 16 Greggio, L., \& Renzini, A. 1983, A\&A, 118, 217

Hernquist, L. 1990, ApJ, 356, 359

Hernquist, L., \& Katz, N. 1989, ApJS, 70, 419

Hjorth, J., \& Madsen, J. 1991, MNRAS, 253, 703

Im, M., Griffiths, R. E., Ratnatunga, K. U., \& Sarajedini, V. L. 1996, ApJ, 461, L79

Katz, N. 1991, ApJ, 368, 325

Katz, N., \& Gunn, J. E. 1991, ApJ, 377, 365

Katz, N., Weinberg, D., \& Hernquist, L. 1996, ApJS, 105, 19

Kauffmann, G., White, S. D. M., \& Guiderdoni, B. 1993, MNRAS, 264, 201

Kawata, D. 1999, Publ. Astron. Soc. Jpn., 51, 931

Kawata, D. 2001a, ApJ, 558, 598

Kawata, D. 2001b, ApJ, 548, 703

Kawata, D., \& Gibson, B. K. 2003, MNRAS, 346, 135

Kobayashi, C. 2005, MNRAS, 361, 1216

Kodama, T., \& Arimoto, N. 1997, A\&A, 320, 41

Kroupa, P. 1998, MNRAS, 298, 231

Larson, R. B. 1975, MNRAS, 173, 671

Lewis, G. F., Babul, A., Katz, N., et al. 2000, ApJ, 536, 623

Lia, C., Portinari, L., \& Carraro, G. 2002, MNRAS, 330, 821

Longhetti, M., Bressan, A., Chiosi, C., \& Rampazzo, R. 2000, A\&A, 353, 917

Macchetto, F., Pastoriza, M., Caon, N., et al. 1996, A\&AS, 120, 463

Madau, P. 2003, in Astronomy, Cosmology and Fundamental Physics, ed. P. A. Shaver, L. Dilella, \& A. Gimenez, 39

Marri, S., \& White, S. D. M. 2003, MNRAS, 345, 561

Merlin, E. 2005, in Formation and evolution of early type galaxies. Models with quasi-cosmological initial conditions, Master Thesis, University of Padova, Italy
Miller, J. M., \& Bertschinger, E. 1986, Bull. Am. Astron. Soc., 18, 927 Monaghan, J. J., \& Lattanzio, J. C. 1985, A\&A, 149, 135

Moore, B., Governato, F., Quinn, T., Stadel, J., \& Lake, G. 1998, ApJ, 499, L5

Navarro, J. F., \& Steinmetz, M. 1997, ApJ, 478, 13

Navarro, J. F., Frenk, C. S., \& White, S. D. M. 1996, ApJ, 462, 563

Padmanabhan, T. 1993, Structure Formation in the Universe, Structure Formation in the Universe (Cambridge, UK: Cambridge University Press)

Patton, D. R., Pritchet, C. J., Yee, H. K. C., Ellingson, E., \& Carlberg, R. G. 1997, ApJ, 475, 29

Peebles, P. J. E. 2002, in A New Era in Cosmology, ASP Conf. Ser., 283, 351

Portinari, L., Chiosi, C., \& Bressan, A. 1998, A\&A, 334, 505

Portinari, L., Sommer-Larsen, J., \& Tantalo, R. 2004, MNRAS, 347, 691

Power, C., Navarro, J. F., Jenkins, A., et al. 2003, MNRAS, 338, 14

Salpeter, E. E. 1955, ApJ, 121, 161

Schade, D., Lilly, S. J., Crampton, D., et al. 1999, ApJ, 525, 31

Schmidt, M. 1959, ApJ, 129, 243

Sersic, J. L. 1968, Atlas de galaxias australes (Cordoba, Argentina: Observatorio Astronomico)

Springel, V., \& Hernquist, L. 2003, MNRAS, 339, 289

Steinmetz, M. 1996, MNRAS, 278, 1005

Tantalo, R., \& Chiosi, C. 2002, A\&A, 388, 396

Tantalo, R., \& Chiosi, C. 2004a, MNRAS, 353, 917

Tantalo, R., \& Chiosi, C. 2004b, MNRAS, 353, 405

Tantalo, R., Chiosi, C., Bressan, A., \& Fagotto, F. 1996, A\&A, 311, 361

Tantalo, R., Chiosi, C., Bressan, A., Marigo, P., \& Portinari, L. 1998, A\&A, 335, 823

Thornton, K., Gaudlitz, M., Janka, H.-T., \& Steinmetz, M. 1998, ApJ, 500, 95 van Dokkum, P. G. 2005, AJ, 130, 2647

White, S. D. M. 1984, ApJ, 286, 38

White, S. D. M., \& Rees, M. J. 1978, MNRAS, 183, 341 